\title{
NEARBY DEBRIS DISK SYSTEMS WITH HIGH FRACTIONAL LUMINOSITY RECONSIDERED
}

\author{
A. Moór, ${ }^{1}$ P. Ábrahám, ${ }^{1}$ A. Derekas, ${ }^{2,3}$ Cs. Kiss, ${ }^{1}$ L. L. Kiss, ${ }^{2}$ D. Apai, ${ }^{4,5}$ C. Grady, ${ }^{6,7}$ and Th. Henning ${ }^{8}$ \\ Received 2005 December 2; accepted 2006 February 8
}

\begin{abstract}
By searching the IRAS and ISO databases, we compiled a list of 60 debris disks that exhibit the highest fractional luminosity values $\left(f_{d}>10^{-4}\right)$ in the vicinity of the Sun $(d<120 \mathrm{pc})$. Eleven out of these 60 systems are new discoveries. Special care was taken to exclude bogus disks from the sample. We computed the fractional luminosity values using available IRAS, ISO, and Spitzer data and analyzed the Galactic space velocities of the objects. The results revealed that stars with disks of high fractional luminosity often belong to young stellar kinematic groups, providing an opportunity to obtain improved age estimates for these systems. We found that practically all disks with $f_{d}>5 \times 10^{-4}$ are younger than $100 \mathrm{Myr}$. The distribution of the disks in the fractional luminosity versus age diagram indicates that (1) the number of old systems with high $f_{d}$ is lower than was claimed before, (2) there exist many relatively young disks of moderate fractional luminosity, and (3) comparing the observations with a current theoretical model of debris disk evolution, a general good agreement could be found.
\end{abstract}

Subject headings: circumstellar matter — infrared: stars — stars: kinematics

\section{INTRODUCTION}

One of the major discoveries of the IRAS mission was that main-sequence stars may exhibit excess emission at infrared (IR) wavelengths ("Vega phenomenon;" Aumann et al. 1984). Systematic searches in the IRAS catalogs (Backman \& Paresce 1993; Mannings \& Barlow 1998; Silverstone 2000; Zuckerman \& Song 2004a and references therein) revealed that $\sim 15 \%$ of main-sequence stars show infrared excess (Plets \& Vynckier 1999). It was suggested already after the first discovery that the excess can be attributed to thermal emission of dust confined into a circumstellar disk (Aumann et al. 1984). The existence of such debris disks was first confirmed by the coronagraphic observation of scattered light from the $\beta$ Pic system (Smith \& Terrile 1984). Subsequent imaging of specific systems at mid-infrared (e.g., HR 4796A; Koerner et al. 1998) and submillimeter wavelengths (e.g., $\epsilon$ Eri; Holland et al. 1998) supported this picture.

The possibility that debris disks might evolve over time was first mentioned by Backman \& Gillett (1987), who proposed that disks around Vega, $\epsilon$ Eridani, and Fomalhaut could be more evolved analogs of the $\beta$ Pic system. Using submillimeter measurements of the best known Vega-like stars, Holland et al. (1998) showed that the derived dust mass of the disks decays with stellar age as a power law. Subsequent studies with the Infrared Space Observatory (ISO; Kessler et al. 1996) demonstrated that debris disks are more common around young stars $(t<400 \mathrm{Myr})$ than around old ones and there is a trend for older debris disks to be less

\footnotetext{
1 Konkoly Observatory of the Hungarian Academy of Sciences, P.O. Box 67, H-1525 Budapest, Hungary.

2 School of Physics A28, University of Sydney, Sydney NSW 2006, Australia.

${ }^{3}$ School of Physics, Department of Astrophysics and Optics, University of New South Wales, Sydney NSW 2052, Australia.

4 Steward Observatory, University of Arizona, 933 North Cherry Avenue, Tucson, AZ 85721.

5 NASA Astrobiology Institute.

6 Eureka Scientific, 2452 Delmer Street Suite 100, Oakland, CA 94602-3017.

7 Exo-Planets and Stellar Astrophysics Laboratory, Exploration of the Universe Division, NASA Goddard Space Flight Center, Code 667, Greenbelt, MD 20771.

8 Max-Planck-Institut für Astronomie, Königstuhl 17, D-69117 Heidelberg, Germany.
}

massive than younger ones (Habing et al. 2001; Spangler et al. 2001; Silverstone 2000). The evolutionary picture was further refined by Decin et al. (2003), who reinvestigated the ISO results and revised the stellar age estimates. Recently Rieke et al. (2005) presented a survey of A-type stars, performed at $24 \mu \mathrm{m}$ with the Multiband Imaging Photometer for Spitzer (MIPS; Rieke et al. 2004) on board the Spitzer Space Telescope (Werner et al. 2004), and observed a general decay on a timescale of $150 \mathrm{Myr}$.

A new generation of theoretical models has been developed to explain the temporal evolution of debris disks (Kenyon \& Bromley 2002; Dominik \& Decin 2003; Kenyon \& Bromley 2004). These models take into account the fact that the destruction timescales of dust grains orbiting main-sequence stars are significantly shorter than the age of the central star; therefore, the observed dust grains in a debris disk must be continuously replenished (Backman \& Paresce 1993). Collisional erosion of minor bodies in exosolar analogs of our solar system and the sublimation of comets are the best explanations for the replenishment process (Harper et al. 1984; Backman \& Paresce 1993). Most recent models link the temporal evolution of debris disks to the formation and erosion of planetesimals (Kenyon \& Bromley 2002, 2004).

The amount of dust in a debris disk is usually characterized by its fractional luminosity, $f_{d}$, defined as the ratio of integrated infrared excess of the disk to the bolometric luminosity of the star. Despite the general evolutionary trend described above, fractional luminosity values of individual systems were found to show a large spread of $10^{-6} \lesssim f_{d} \lesssim 10^{-3}$ at almost any age (Decin et al. 2003). Rieke et al. (2005) also found large variations of the IR excess around A-type stars within each age group. They emphasized the role of individual collisional events between large planetesimals as one of the possible explanations of their result.

Particularly interesting is the relatively high number of older systems $(t \gtrsim 500 \mathrm{Myr})$ with high fractional luminosity values $\left(f_{d} \simeq 10^{-3}\right)$. These systems pose a serious challenge even to the new generation of theoretical models. A qualitative explanation for the existence of debris disks with large $f_{d}$ values around old stars was suggested by Dominik \& Decin (2003). According to their theory, different planetesimal disks become active debris systems at different ages because of the delayed onset of collisional cascades. 
The presence of relatively old systems with high fractional luminosity, however, might partly be an observational artifact, since several factors could bias the distribution of debris disks on the $f_{d}$ versus age diagram. Some stars have been nominated as Vega candidates due to erroneous infrared photometric measurements, confusion by background sources, or the presence of extended nebulosity (where the IR emission is of interstellar rather than of circumstellar origin). In order to obtain a reliable picture of debris disk evolution, these misidentifications ("bogus disks") have to be found and discarded. The actual positions of confirmed debris disks in the $f_{d}$ versus age diagram might also be biased by measurement errors in the far-infrared photometry and even more significantly uncertainties in the age determination.

In this paper we study a sample of debris disks that exhibit the highest fractional luminosity values in the solar neighborhood. Setting a threshold value of $f_{d}=10^{-4}$ and a distance limit of $120 \mathrm{pc}$, we compiled a list of 60 disks and performed an accurate determination of their infrared excess using IRAS, ISO, and Spitzer data ( $\S 2)$. In $\S 3$ we present improved age estimates for a large number of stars, derived, e.g., by determining their membership in young moving groups. In $\S 4$ we analyze the distribution of the disks in the $f_{d}$ versus age diagram and find a significant fraction of our sample to be younger than was previously thought.

Our conclusions are summarized in $\S 5$. In Appendix A we list bogus disks identified in our work. Appendix B lists new members of young moving groups discovered in the present study.

\section{SAMPLE SELECTION}

We created the input list for this study by (1) identifying debris disk candidates in the IRAS and ISO databases, (2) rejecting bogus debris disks and suspicious objects, and (3) computing infrared fractional luminosity values and selecting disks with $f_{d}>10^{-4}$.

\subsection{Searching the IRAS Catalogs for Stars with Infrared Excess}

With the aim of compiling a list of main-sequence stars with IR excess, we made a systematic search in the IRAS Faint Source Survey Catalog (FSC; Moshir et al. 1989) and in the IRAS Serendipitous Survey Catalog (SSC; Kleinmann et al. 1986). In order to reduce source confusion, our survey was confined to $|b| \geq 10^{\circ}$ Galactic latitudes. We selected all infrared sources with at least moderate flux quality at 25 or $60 \mu \mathrm{m}$, and their positions were correlated with entries from the Hipparcos Catalogue (ESA 1997) and the Tycho-2 Spectral Type Catalogue (Wright et al. 2003). Positional coincidences within $30^{\prime \prime}$ were extracted. In order to assure that the selected objects are not giant stars, the luminosity class was constrained to IV-V in the Hipparcos catalog and to $\mathrm{V}$ in the Tycho catalog. We also included several objects whose luminosity class was not available in the Hipparcos catalog but whose absolute magnitudes indicated a main-sequence evolutionary phase. Since the infrared excess from early B-type stars might be due to free-free emission (Zuckerman 2001), the sample was limited to spectral types later than B9 in both catalogs. Our query is similar to that of Silverstone (2000), but since we also considered the IRAS $25 \mu \mathrm{m}$ band, it is also sensitive to stars with excess from warmer dust disk.

Following the principles of the method by Plets \& Vynckier (1999), for each selected star we predicted the far-infrared flux density of the stellar photosphere using the $K_{s}$-band magnitude (or $V$ band, when good quality $K_{s}$ photometry was not available) and the $B-V$ color index. $K_{s}$-band photometry was drawn from the Two Micron All Sky Survey (2MASS) catalog (Cutri et al.
2003), and $V$ magnitudes and $B-V$ color indices were taken from the Hipparcos and Tycho catalogs. As a first step a photospheric $25 \mu \mathrm{m}$ flux density was derived from the $K_{s}$ magnitude and the $B-V$ color of the star using the collection of stellar model predictions by M. Cohen and P. Hammersley (available on the ISO Data Centre Web site ${ }^{9}$ ). Then, color relationships predicting the photospheric flux ratios between $25 \mu \mathrm{m}$ and a selection of IRAS, ISO, and Spitzer photometric bands were also derived from the same stellar models. The average accuracy of the predicted far-infrared fluxes is estimated to be around $4 \%$ when computed from the $K_{s}$ magnitudes and $8 \%$ when computed from $V$ magnitudes.

In order to compute IR excess values, the predicted photospheric flux densities were subtracted from the measured flux densities in each IRAS band. In principle the IRAS fluxes have to be color corrected since the shape of the spectral energy distribution of the system usually differs from the $F_{\nu} \sim \nu^{-1}$ reference spectrum (this spectral shape was assumed while the flux densities quoted in the IRAS catalogs were derived from the detector in-band powers). Since the true spectrum of the system is not known a priori, we decided to multiply the predicted photospheric fluxes (rather than dividing the IRAS flux densities) with color correction factors appropriate for a stellar photosphere (Beichman et al. 1988). The significance level of the infrared excess was calculated in each photometric band with the following formula:

$$
S_{\text {excess }}=\frac{F_{\text {meas }}-F_{\text {pred }}}{\sqrt{\delta F_{\text {meas }}^{2}+\delta F_{\text {pred }}^{2}}},
$$

where $\delta F_{\text {meas }}$ is the quoted uncertainty in the FSC or SSC and $\delta F_{\text {pred }}$ is the uncertainty of the prediction described above. When $S_{\text {excess }}$ was greater than 3 either in the 25 or $60 \mu \mathrm{m}$ bands, the object was selected as an excess candidate star. Applying the above criteria, we identified in total 355 excess candidate stars in the IRAS databases.

\subsection{ISO-based Selection of Stars with Infrared Excesses}

In a second step the IRAS-based list was supplemented with excess stars selected from the $I S O$ databases. The Vega phenomenon was a key program for $I S O$ (see $\S 1$ ), and a number of stars have been observed with ISOPHOT, the onboard photometer (Lemke et al. 1996). We collected all ISOPHOT observations of normal stars from different observers performed in minimap, sparse map, or staring mode (a detailed description of these observing modes is given in Laureijs et al. 2003) and performed a homogeneous reevaluation of the whole sample (Ábrahám et al. 2003; P. Ábrahám et al. 2006, in preparation). For details of the data analysis and criteria for candidate excess stars, see P. Ábrahám et al. (2006, in preparation). We note that most selected candidates have already been published by the original observers (Decin et al. 2000; Habing et al. 2001; Spangler et al. 2001; Silverstone 2000), but due to their different processing schemes, the published flux densities cannot be directly merged for a homogeneous catalog. The merged IRAS- and ISO-based list includes altogether 364 IR excess stars.

\subsection{Rejection of Suspicious Objects}

Since our goal is to compile a list of debris disks, we excluded all known young stellar objects (e.g., T Tauri or Herbig Ae/Be stars) that harbor protoplanetary disks. The sample could also be contaminated by source confusion: due to the low spatial

\footnotetext{
9 Available at http://www.iso.vilspa.esa.es/users/expl_lib/ISO/wwwcal.
} 
resolution of IRAS at far-IR wavelengths, many of the positional coincidences between a star and a far-IR source could be bogus and the far-IR emission is related to a foreground or background object.

For part of the sample (110 stars) higher spatial resolution infrared maps are available, obtained by either the ISOPHOT or MIPS instrument. We downloaded ISOPHOT data from the ISO Data Archive (IDA) and processed with the Phot Interactive Analysis (PIA) version 10.0 (Gabriel et al. 1997). MIPS basic calibrated data (BCD) files were downloaded from the Spitzer Science Center data archive. The latter products are composed of two-dimensional FITS image files that included all general calibrations and corrections for MIPS detectors (Gordon et al. 2005). In each case these data were co-added and corrected for array distortions with the Spitzer Science Center MOPEX (MOsaicking and Point source EXtraction; Makovoz \& Marleau 2005) software. Bad data flagged in the BCD mask files, as well as permanently damaged pixels flagged in the static pixel mask file, were ignored during the data combination. Output mosaics had pixels with size of 2.5 at $24 \mu \mathrm{m}$ and $4^{\prime \prime}$ at $70 \mu \mathrm{m}$. The MOPEX/APEX software package was used to detect sources and determine their positions on the final maps.

The positions of the infrared sources were determined on the ISO and Spitzer maps, and objects whose coordinates differed from the optical position (and in some cases coincided with a nearby background object) and/or associated with extended nebulosity were discarded from the list. For positional discrepancy the threshold value was set to half of the width of a point source's footprint. In the case of ISOPHOT the large pixel size dominated the footprint, and in the 60-100 $\mu \mathrm{m}$ range the threshold was $23^{\prime \prime}$. In the case of MIPS arrays the footprint was defined by the telescope point-spread function, and we adopted threshold values of $3^{\prime \prime}$ and $9^{\prime \prime}$ at 24 and $70 \mu \mathrm{m}$, respectively. The absolute pointing uncertainty was less than these values for both satellites. In total 24 disk candidates were dropped from the list.

When neither ISO nor Spitzer maps were available, we made an attempt to filter out bogus disks by assuming that an object is possibly affected by source confusion if one of the following applies:

1. A known galaxy or evolved star $(\mathrm{OH} / \mathrm{IR}$ source, Mira variable) is located within $1^{\prime}$ of the IRAS position.

2. A source included in the IRAS Small Scale Structure Catalog (Helou \& Walker 1988) or in the 2MASS Extended Source Catalog (Jarrett et al. 2000) is located within $1^{\prime}$ of the IRAS position.

3. A 2MASS source with an excess in the $K_{s}$ band (identified in the $H-K_{s}$ vs. $J-H$ diagram in comparison with the locus of the main sequence and taking into account the reddening path) is located within $1^{\prime}$ of the IRAS position.

4. The $60 \mu \mathrm{m} / 100 \mu \mathrm{m}$ flux ratio of the candidate source resembles the color of infrared cirrus $\left(F_{60} / F_{100}<0.25\right.$, which corresponds to blackbody temperatures lower than $33 \mathrm{~K}$ ). At least moderate flux quality flags were required in both IRAS bands.

These cases were also discarded from our list of debris disk candidates (48 objects).

In an earlier study, Kalas et al. (2002) used high angular resolution coronagraphic observations at optical wavelengths and found cases where the far-infrared excess observed by IRAS was of interstellar (rather than circumstellar) origin ("Pleiades phenomenon"), leading to false entries in the Vega candidate lists. They also suggested that a significant fraction of Vega candidates beyond the Local Bubble might be bogus, since the star illuminates nearby interstellar matter rather than a circumstellar disk.
After checking the positions of our sources projected on recent maps of the Local Bubble (Lallement et al. 2003), we discarded all objects situated in the wall of the bubble or beyond. The wall was defined as the isocontours corresponding to the $50 \mathrm{~m} \AA \mathrm{Na}$ I D2 line equivalent widths in the maps. In practice nearly all of our sources beyond $120 \mathrm{pc}$ were removed, while within this radius only a few were dropped. Thus, we defined a maximum distance limit of 120 pc for our stars, constructing a nearly complete volume-limited sample.

\subsection{The List of Disks with High Fractional Luminosity}

In order to compute fractional luminosity values for each candidate star, we constructed spectral energy distributions by combining infrared fluxes from the FSC, SSC, and ISOPHOT (reevaluated by us; see P. Ábrahám et al. 2006, in preparation) and additional MIPS and submillimeter fluxes from the literature. The excess above the predicted photosphere was fitted by a single-temperature modified blackbody, where the emissivity was assumed to vary as $1-\exp \left[-\left(\lambda_{0} / \lambda\right)^{\beta}\right]$, where $\lambda_{0}$ was set to $100 \mu \mathrm{m}$ (see, e.g., Williams et al. 2004). We fixed $\beta$ equal to 1 , which is a typical value in the case of debris systems (Dent et al. 2000). If the excess was detected at one wavelength only, we adopted a modified blackbody whose peak (in $F_{\nu}$ ) coincided with that single wavelength. From the fitted spectral shape color correction factors were computed and applied to the data. Then again a modified blackbody was fitted, resulting in new color correction factors, and this procedure was repeated until the color correction factors converged. Finally, the fractional dust luminosity was calculated as $f_{d}=L_{\mathrm{IR}} / L_{\mathrm{bol}}$. In order to estimate the uncertainties on our fractional luminosity values, we performed a Monte Carlo simulation. We added Gaussian noise to the photometric data points using their quoted $1 \sigma$ photometric errors and then recomputed the fractional dust luminosities. Formal uncertainties of the predicted theoretical photospheric fluxes were also taken into account. Final uncertainties were derived as the standard deviation of these values after 1000 repetitions. We note that these values include only random uncertainties; systematic errors due to, e.g., limited wavelength coverage are not taken into account.

Artymowicz (1996) argued that debris disks are confined to $f_{d}<10^{-2}$ and sources with higher fractional luminosity probably contain a significant amount of gas (e.g., T Tauri and Herbig Ae/Be stars, "transition" objects). Therefore, we excluded objects with $f_{d}>10^{-2}$ from our sample. Then the remaining sample was sorted by decreasing $f_{d}$ values and stars with $f_{d}>10^{-4}$ (60 stars) were taken for the further analysis presented in this paper.

Basic stellar parameters for these 60 objects, as well as derived fractional luminosities and their uncertainties, are presented in Table 1. Infrared data used in our analysis, including both the original flux values as listed in the catalogs or provided by our reduction algorithm and corrected fluxes, where color correction was applied, are given in Table 2. The table also contains photospheric flux predictions for the specific wavelengths. Inspecting the flux density values obtained by different instruments at the same wavelength (e.g., IRAS and ISOPHOT at $60 \mu \mathrm{m}$ ), one finds discrepancies that may arise, e.g., from the different beams and different calibration strategies of the instruments. Comparing the IRAS and ISOPHOT flux values in Table 2, a general good agreement within $1 \sigma$ was found, with no deviations above the $3 \sigma$ limit (the uncertainty $\sigma$ was computed as the quadratic sum of quoted uncertainties from the two instruments). We also compared our ISOPHOT flux densities with the results of earlier evaluations of the same observations in the literature. In most cases the results were consistent (except HD 10647 and HD 53143, where 
TABLE 1

Stellar Properties and Derived Fractional Luminosities of the Disks

\begin{tabular}{|c|c|c|c|c|c|c|c|c|c|c|c|}
\hline $\begin{array}{l}\text { Name } \\
(1)\end{array}$ & $\begin{array}{l}\text { Other Name } \\
\text { (2) }\end{array}$ & $\begin{array}{c}\text { Spectral Type } \\
\text { (3) }\end{array}$ & $\begin{array}{c}V \\
(\mathrm{mag}) \\
(4)\end{array}$ & $\begin{array}{c}B-V \\
(\mathrm{mag}) \\
(5)\end{array}$ & $\begin{array}{l}\text { Distance } \\
(\mathrm{pc}) \\
(6)\end{array}$ & $\begin{array}{l}\text { First Reference to } \\
\text { Debris Disk } \\
\text { (7) }\end{array}$ & $\begin{array}{l}\text { Debris Disk } \\
\text { Confirmation } \\
\text { (8) }\end{array}$ & $\begin{array}{l}\text { References } \\
\text { (9) }\end{array}$ & $\begin{array}{c}f_{d} \\
\left(10^{-4}\right) \\
(10)\end{array}$ & $\begin{array}{l}\text { Age } \\
\text { (Myr) } \\
(11)\end{array}$ & $\begin{array}{c}\text { Age References } \\
\text { (12) }\end{array}$ \\
\hline HD $105 \ldots \ldots \ldots \ldots \ldots . . . . .$. & $\ldots$ & G0 V & 7.51 & 0.595 & 40 & 1 & ISO/MIPS & 1,2 & $2.5 \pm 0.3$ & $30_{-20}^{+10}$ & 30 \\
\hline HD 377 ...................... & $\ldots$ & G2 V & 7.59 & 0.626 & 40 & 2 & MIPS & 2 & $4.0 \pm 0.3$ & {$[30,100]$} & 31,32 \\
\hline 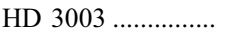 & $\ldots$ & A0 V & 5.07 & 0.038 & 46 & 3 & MIPS* & & $1.4 \pm 0.2$ & $30_{-20}^{+10}$ & 30 \\
\hline 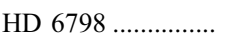 & $\ldots$ & A3 V & 5.60 & 0.008 & 83 & 4 & IRS & 12 & $1.6 \pm 0.3$ & $340_{-80}^{+60}$ & 7 \\
\hline HD 8907 ................... & $\ldots$ & F8 & 6.66 & 0.505 & 34 & 1 & ISO/MIPS & 1,2 & $2.4 \pm 0.1$ & {$[100,870]$} & $31,33,34$ \\
\hline HD $9672 \ldots \ldots \ldots \ldots \ldots . . . .$. & 49 Cet & A1 V & 5.62 & 0.066 & 61 & 5 & ISO & 13 & $9.2 \pm 0.6$ & {$[8,20]$} & 34,35 \\
\hline HD $10472 \ldots \ldots \ldots \ldots . . . .$. & $\ldots$ & F2 IV/V & 7.62 & 0.420 & 67 & 1 & MIPS* & 14 & $3.4 \pm 0.9$ & {$[20,150]$} & 30 \\
\hline HD 10647 ............... & $\ldots$ & F8 V & 5.52 & 0.551 & 17 & 6 & ISO/MIPS* & 15 & $3.0 \pm 0.3$ & {$[300,7000]$} & 34,36 \\
\hline HD $10638 \ldots \ldots \ldots \ldots . . . .$. & $\ldots$ & A3 & 6.73 & 0.247 & 72 & 1 & & & $3.9 \pm 0.5$ & {$[20,150]$} & 30 \\
\hline HD $15115 \ldots \ldots \ldots \ldots \ldots . . .$. & $\ldots$ & $\mathrm{F} 2$ & 6.79 & 0.399 & 45 & 1 & ISO/MIPS & 1 & $4.9 \pm 0.4$ & $12_{-4}^{+8}$ & 30 \\
\hline HD $15745 \ldots \ldots \ldots \ldots \ldots$ & $\ldots$ & F0 & 7.47 & 0.360 & 64 & 1 & ISO/MIPS & 1 & $20.1 \pm 1.4$ & $<700$ & 34,36 \\
\hline HD $16743 \ldots \ldots \ldots \ldots . . . .$. & $\ldots$ & F0/F2 III/IV & 6.78 & 0.387 & 60 & 7 & MIPS & & $3.6 \pm 0.3$ & $1200_{-600}^{+500}$ & 36 \\
\hline HD $17390 \ldots \ldots \ldots \ldots . . . .$. & $\ldots$ & F3 IV/V & 6.48 & 0.387 & 45 & 1 & ISO/MIPS* & 16 & $1.9 \pm 0.2$ & $<800$ & 34,36 \\
\hline HD 21997 ................ & $\ldots$ & A3 IV/V & 6.38 & 0.120 & 74 & 4 & IRS & 12 & $4.7 \pm 0.3$ & $20_{-10}^{+10}$ & 30 \\
\hline HD $24966 \ldots \ldots \ldots \ldots . . . .$. & $\ldots$ & $\mathrm{A} 0 \mathrm{~V}$ & 6.89 & 0.023 & 104 & 1 & $\ldots$ & & $2.4 \pm 0.5$ & $<100$ & 7 \\
\hline HD $25457 \ldots \ldots \ldots \ldots . . .$. & $\ldots$ & F5 V & 5.38 & 0.516 & 19 & 1 & ISO/MIPS & 1,2 & $1.0 \pm 0.2$ & {$[50,100]$} & 30 \\
\hline HD 30447 .................. & $\ldots$ & F3 V & 7.85 & 0.393 & 78 & 1 & ISO/MIPS & 16 & $7.5 \pm 1.1$ & $20_{-10}^{+10}$ & 30 \\
\hline HD 32297 ................ & $\ldots$ & A0 & 8.13 & 0.199 & 112 & 1 & COR & 17 & $33.4 \pm 6.0$ & $<30$ & 37 \\
\hline HD $35841 \ldots \ldots \ldots \ldots . . .$. & $\ldots$ & F5 V & 8.91 & 0.496 & $104^{\mathrm{P}}$ & 1 & $\ldots$ & & $15.5 \pm 3.7$ & $20_{-10}^{+10}$ & 30 \\
\hline HD $37484 \ldots \ldots \ldots \ldots . . . .$. & $\ldots$ & F3 V & 7.26 & 0.404 & 60 & 4 & ISO/MIPS & 1,2 & $2.7 \pm 0.5$ & $30_{-20}^{+10}$ & 30 \\
\hline HD $38207 \ldots \ldots \ldots \ldots . . . . .$. & $\ldots$ & $\mathrm{F} 2 \mathrm{~V}$ & 8.47 & 0.391 & $103^{\mathrm{P}}$ & 1 & ISO/MIPS & 1,2 & $10.8 \pm 0.6$ & $20_{-10}^{+10}$ & 30 \\
\hline HD $38206 \ldots \ldots \ldots \ldots . . . .$. & $\ldots$ & $\mathrm{A} 0 \mathrm{~V}$ & 5.73 & -0.014 & 69 & 8 & MIPS & 18 & $1.4 \pm 0.3$ & $20_{-10}^{+10}$ & 30 \\
\hline HD $38678 \ldots \ldots \ldots \ldots . . . .$. & $\zeta$ Lep & A2 Vann & 3.55 & 0.104 & 22 & 9 & ISO/MIPS* & 19 & $1.1 \pm 0.2$ & $200_{-100}^{+100}$ & 30 \\
\hline HD $39060 \ldots \ldots \ldots \ldots . . . . .$. & $\beta$ Pic & A3 V & 3.85 & 0.171 & 19 & 10 & ISO/COR/SUBM & $10,20,21$ & $24.3 \pm 1.1$ & $12_{-4}^{+8}$ & 30 \\
\hline HD $50571 \ldots \ldots \ldots \ldots . . . . .$. & $\ldots$ & F7 III-IV & 6.11 & 0.457 & 33 & 7 & MIPS $^{*}$ & & $1.1 \pm 0.3$ & $1800_{-1300}^{+1000}$ & 36 \\
\hline HD $53143 \ldots \ldots \ldots \ldots . . .$. & $\ldots$ & K0 IV-V & 6.81 & 0.786 & 18 & 8 & ISO & 15 & $2.0 \pm 0.5$ & $980_{-330}^{+520}$ & 15 \\
\hline HD $54341 \ldots \ldots \ldots \ldots . . . .$. & $\ldots$ & A0 V & 6.52 & -0.008 & 93 & 7 & & & $1.8 \pm 0.4$ & $<100$ & 7 \\
\hline HD $69830 \ldots \ldots \ldots . . . .$. & $\ldots$ & $\mathrm{K} 0 \mathrm{~V}$ & 5.95 & 0.754 & 13 & 8 & IRS & 22 & $2.0^{\mathrm{a}}$ & {$[600,4700]$} & 3,33 \\
\hline HD $76582 \ldots \ldots \ldots \ldots . . .$. & $\ldots$ & F0 IV & 5.68 & 0.209 & 49 & 1 & $\ldots$ & & $1.7 \pm 0.2$ & $450_{-290}^{+150}$ & 7 \\
\hline HD $78702 \ldots \ldots \ldots \ldots . . . .$. & $\ldots$ & $\mathrm{A} 0 / \mathrm{A} 1 \mathrm{~V}$ & 5.73 & 0.000 & 80 & 1 & $\ldots$ & & $2.1 \pm 0.7$ & $220_{-140}^{+100}$ & 7 \\
\hline HD 84870A.............. & $\ldots$ & A3 & 7.20 & 0.233 & 90 & 1 & $\ldots$ & & $4.5 \pm 1.0$ & $<520$ & 7 \\
\hline HD $85672 \ldots \ldots \ldots \ldots . .$. & $\ldots$ & A0 & 7.59 & 0.159 & 93 & 1 & $\cdots$ & & $4.9 \pm 1.0$ & $<100$ & 7 \\
\hline HD $92945 \ldots \ldots \ldots \ldots . . . .$. & $\ldots$ & $\mathrm{K} 1 \mathrm{~V}$ & 7.72 & 0.873 & 22 & 1 & MIPS & 23 & $5.3 \pm 1.2$ & 100 & 32,33 \\
\hline HD $107146 \ldots \ldots \ldots \ldots$ & $\ldots$ & $\mathrm{G} 2 \mathrm{~V}$ & 7.04 & 0.604 & 29 & 1 & MIPS/COR/SUBM & $2,24,25$ & $9.2 \pm 0.9$ & $100_{-20}^{+100}$ & 25,34 \\
\hline HD 109573A........... & HR 4796A & $\mathrm{A} 0 \mathrm{~V}$ & 5.78 & 0.003 & 67 & 11 & $\mathrm{MIR} / \mathrm{COR}$ & 26,27 & $47.0 \pm 2.5$ & $8_{-3}^{+7}$ & 30 \\
\hline HD $110058 \ldots \ldots \ldots \ldots . . . .$. & $\ldots$ & $\mathrm{A} 0 \mathrm{~V}$ & 7.99 & 0.148 & 100 & 8 & ISO & 16 & $18.9 \pm 3.3$ & $<100$ & 7 \\
\hline HD $115116 \ldots \ldots \ldots \ldots . . . .$. & $\ldots$ & A7 V & 7.07 & 0.205 & 85 & 7 & $\ldots$ & & $3.2 \pm 1.0$ & $<360$ & 7 \\
\hline HD $120534 \ldots \ldots \ldots . . .$. & $\ldots$ & A5 V & 7.02 & 0.275 & $67^{\mathrm{P}}$ & 1 & $\ldots$ & & $4.9 \pm 0.9$ & $<320$ & 7 \\
\hline HD $121812 \ldots \ldots \ldots . .$. & $\ldots$ & K0 & 8.53 & 0.820 & 38 & 7 & $\ldots$ & & $14.9 \pm 4.1$ & $230_{-90}^{+150}$ & 7 \\
\hline HD $122106 \ldots \ldots \ldots \ldots$ & $\ldots$ & F8 V & 6.36 & 0.486 & 78 & 7 & $\ldots$ & & $1.2 \pm 0.4$ & {$[1000,1600]$} & 36,38 \\
\hline HD $127821 \ldots \ldots \ldots \ldots$ & $\ldots$ & F4 IV & 6.10 & 0.428 & 32 & 1 & MIPS* & & $2.2 \pm 0.4$ & {$[200,3400]$} & 34,36 \\
\hline HD $130693 \ldots \ldots \ldots . .$. & $\ldots$ & G6 V & 8.20 & 0.734 & $33^{P}$ & 7 & $\ldots$ & & $5.8 \pm 1.9$ & $50_{-40}^{+50}$ & 7 \\
\hline HD $131835 \ldots \ldots \ldots . .$. & $\ldots$ & A2 IV & 7.88 & 0.192 & 111 & 7 & $\ldots$ & & $19.9 \pm 3.3$ & $17_{-1}^{+1}$ & 30 \\
\hline HD 157728 ............. & $\ldots$ & F0 IV & 5.70 & 0.229 & 43 & 6 & $\ldots$ & & $2.9 \pm 0.4$ & $<200$ & 7 \\
\hline HD $158352 \ldots \ldots \ldots \ldots$ & $\ldots$ & A8 V & 5.41 & 0.237 & 63 & 9 & $\ldots$ & & $1.8 \pm 0.5$ & $750_{-150}^{+150}$ & 7 \\
\hline HD 164249 .............. & $\ldots$ & F5 V & 7.01 & 0.458 & 47 & 1 & ISO/MIPS* & 1 & $10.4 \pm 1.6$ & $12_{-4}^{+8}$ & 30 \\
\hline
\end{tabular}


TABLE 1-Continued

\begin{tabular}{|c|c|c|c|c|c|c|c|c|c|c|c|}
\hline $\begin{array}{l}\text { Name } \\
(1)\end{array}$ & $\begin{array}{l}\text { Other Name } \\
\text { (2) }\end{array}$ & $\begin{array}{l}\text { Spectral Type } \\
\text { (3) }\end{array}$ & $\begin{array}{c}V \\
(\mathrm{mag}) \\
(4)\end{array}$ & $\begin{array}{c}B-V \\
(\mathrm{mag}) \\
(5)\end{array}$ & $\begin{array}{l}\text { Distance } \\
\text { (pc) } \\
(6)\end{array}$ & $\begin{array}{l}\text { First Reference to } \\
\text { Debris Disk } \\
\text { (7) }\end{array}$ & $\begin{array}{c}\text { Debris Disk } \\
\text { Confirmation } \\
\text { (8) }\end{array}$ & $\begin{array}{l}\text { References } \\
\text { (9) }\end{array}$ & $\begin{array}{c}f_{d} \\
\left(10^{-4}\right) \\
(10)\end{array}$ & $\begin{array}{l}\text { Age } \\
\text { (Myr) } \\
(11)\end{array}$ & $\begin{array}{c}\text { Age References } \\
\text { (12) }\end{array}$ \\
\hline HD $169666 \ldots \ldots \ldots \ldots . . . . .$. & $\ldots$ & F5 & 6.68 & 0.444 & 51 & 7 & MIPS & & $1.5 \pm 0.3$ & $2200_{-600}^{+400}$ & 36 \\
\hline HD $170773 \ldots \ldots \ldots \ldots$ & $\ldots$ & F5 V & 6.22 & 0.429 & 36 & 5 & ISO/MIPS* & 1 & $3.8 \pm 0.4$ & {$[200,2800]$} & 34,36 \\
\hline 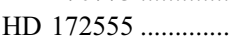 & & A7 V & 4.78 & 0.199 & 29 & 9 & MIPS* & & $7.8 \pm 0.7$ & $12_{-4}^{+8}$ & 30 \\
\hline HD $181296 \ldots \ldots \ldots \ldots$ & $\eta \mathrm{Tel}$ & A0 Vn & 5.03 & 0.020 & 48 & 8 & ISO/MIPS* & 1 & $2.4 \pm 0.2$ & $12_{-4}^{+8}$ & 30 \\
\hline 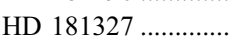 & & F5/F6 V & 7.04 & 0.480 & 51 & 8 & ISO/MIPS* & 16 & $29.3 \pm 1.6$ & $12_{-4}^{+8}$ & 30 \\
\hline HD $182681 \ldots \ldots \ldots \ldots . . .$. & $\ldots$ & $\mathrm{B} 8 / \mathrm{B} 9 \mathrm{~V}$ & 5.66 & -0.014 & 69 & 7 & $\ldots$ & & $1.5 \pm 0.3$ & $<100$ & 7 \\
\hline 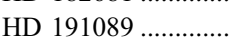 & $\ldots$ & F5 V & 7.18 & 0.480 & 54 & 8 & ISO/MIPS* & 16 & $19.1 \pm 2.2$ & $12_{-4}^{+8}$ & 30 \\
\hline HD $192758 \ldots \ldots \ldots \ldots . . . . .$. & $\ldots$ & $\mathrm{F} 0 \mathrm{~V}$ & 7.02 & 0.317 & $62^{\mathrm{P}}$ & 1 & ISO/MIPS & 16 & $5.6 \pm 0.5$ & {$[35,55]$} & 30 \\
\hline HD $197481 \ldots \ldots \ldots \ldots . . .$. & AU Mic & M1 Ve & 8.81 & 1.470 & 10 & 1 & MIPS/COR/SUBM & $23,28,29$ & $4.0 \pm 0.3$ & $12_{-4}^{+8}$ & 30 \\
\hline 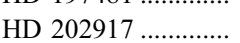 & $\ldots$ & G5 V & 8.65 & 0.690 & 46 & 1 & MIPS* & 1 & $2.9 \pm 0.8$ & $30_{-20}^{+10}$ & 30 \\
\hline HD $205674 \ldots \ldots \ldots \ldots \ldots$ & $\cdots$ & F3/F5 IV & 7.19 & 0.396 & 53 & 7 & MIPS* & & $3.5 \pm 0.8$ & $2600_{-1400}^{+900}$ & 36 \\
\hline 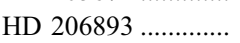 & $\ldots$ & F5 V & 6.69 & 0.439 & 39 & 1 & ISO/MIPS* & 1 & $2.3 \pm 0.2$ & $<2800$ & 34,36 \\
\hline HD $218396 \ldots \ldots \ldots \ldots . . .$. & $\ldots$ & A5 V & 5.97 & 0.259 & 40 & 1 & ISO & 1 & $2.3 \pm 0.2$ & {$[20,150]$} & 30 \\
\hline HD $221853 \ldots \ldots \ldots \ldots . . . .$. & $\ldots$ & F0 & 7.35 & 0.405 & 71 & 1 & ISO/MIPS & 1 & $8.0 \pm 1.1$ & {$[20,150]$} & 30 \\
\hline
\end{tabular}

Notes.-Col. (1): Names. Col. (2): Other names. Cols. (3)-(5): Data are from the Hipparcos or the Tycho-2 Spectral Type Catalog. Col. (6): Distances. P indicates photometric distances; otherwise, Hipparcos distances

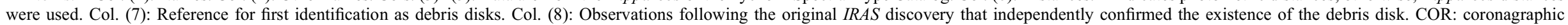

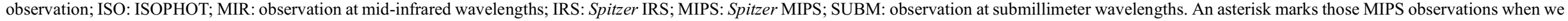

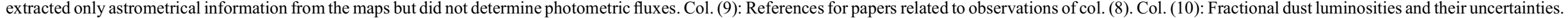
Col. (11): Ages estimates; [t1, t2] marks an age interval. Col. (12): References for age estimates.

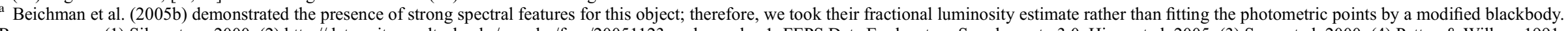

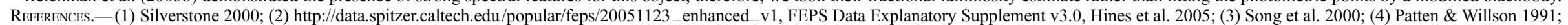

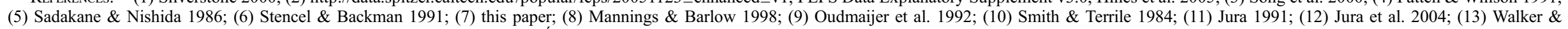

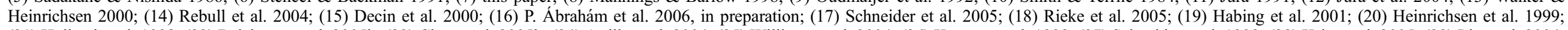

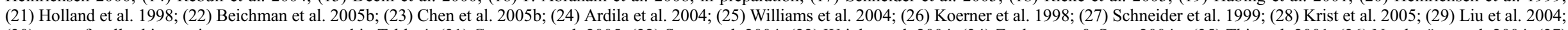

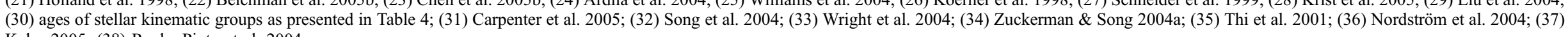
Kalas 2005; (38) Rocha-Pinto et al. 2004. 
TABLE 2

Summary Table of Fluxes Measured in Excess

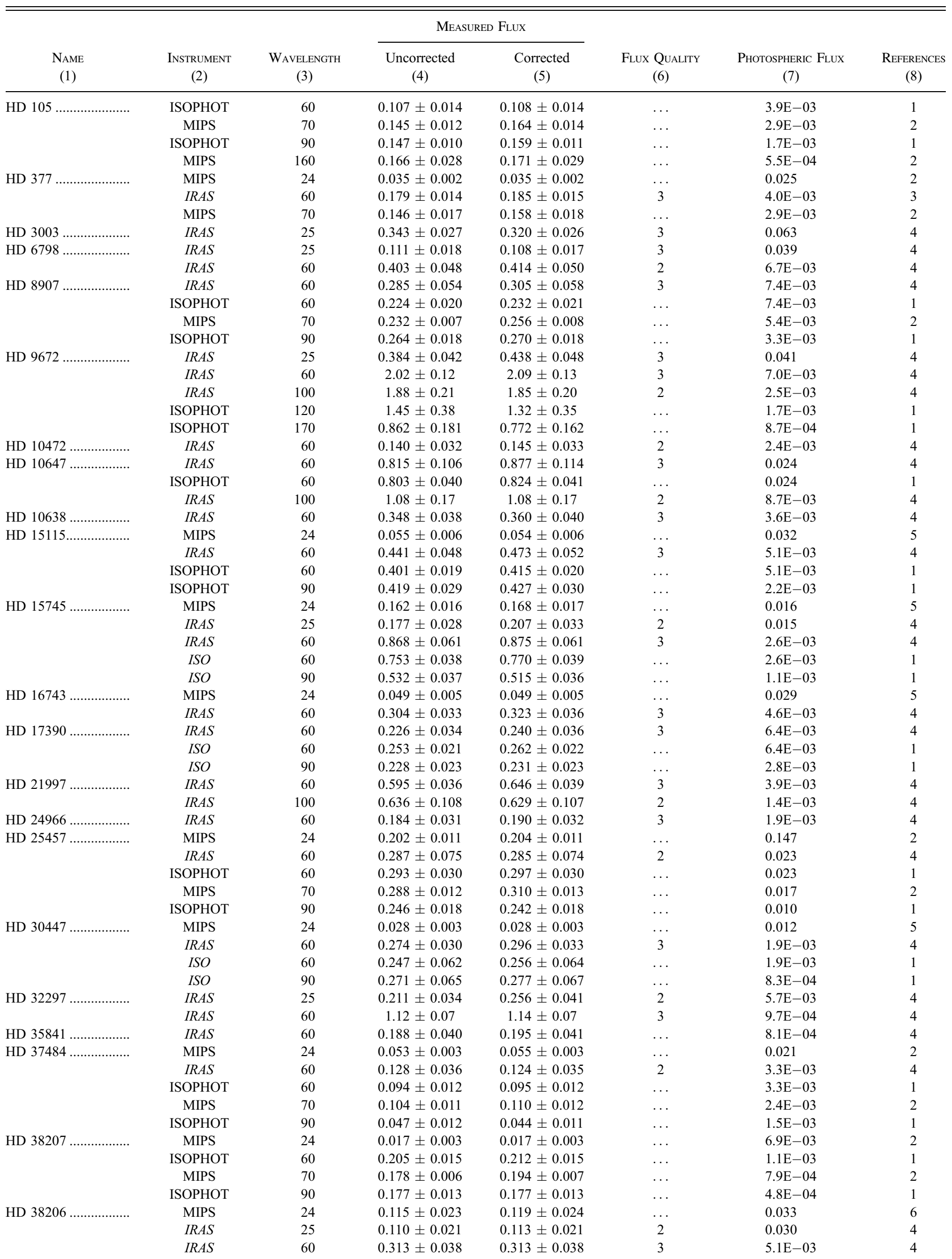


TABLE 2-Continued

\begin{tabular}{|c|c|c|c|c|c|c|c|}
\hline $\begin{array}{l}\text { NAME } \\
(1)\end{array}$ & $\begin{array}{l}\text { INSTRUMENT } \\
\text { (2) }\end{array}$ & $\begin{array}{l}\text { WAVELENGTH } \\
\text { (3) }\end{array}$ & \multicolumn{2}{|c|}{ Measured Flux } & $\begin{array}{c}\text { Flux Quality } \\
\text { (6) }\end{array}$ & $\begin{array}{c}\text { PhOTOSPHERIC FLuX } \\
\text { (7) }\end{array}$ & $\begin{array}{c}\text { ReFERENCES } \\
\text { (8) }\end{array}$ \\
\hline \multirow{2}{*}{ 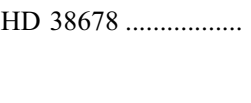 } & IRAS & 60 & $0.366 \pm 0.073$ & $0.302 \pm 0.060$ & 3 & 0.051 & 4 \\
\hline & ISOPHOT & 60 & $0.404 \pm 0.020$ & $0.390 \pm 0.019$ & $\ldots$ & 0.051 & 1 \\
\hline \multirow[t]{6}{*}{ 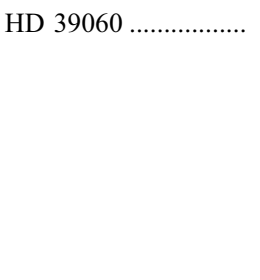 } & $I R A S$ & 12 & $3.40 \pm 0.14$ & $2.38 \pm 0.10$ & 3 & 1.09 & 4 \\
\hline & IRAS & 100 & $11.0 \pm 0.44$ & $9.32 \pm 0.37$ & 3 & 0.015 & 4 \\
\hline & ISOPHOT & 120 & $7.9 \pm 1.2$ & $6.68 \pm 1.01$ & $\ldots$ & 0.011 & 1 \\
\hline & ISOPHOT & 150 & $4.80 \pm 0.73$ & $4.39 \pm 0.67$ & $\ldots$ & $6.8 \mathrm{E}-03$ & 1 \\
\hline & ISOPHOT & 170 & $4.0 \pm 0.6$ & $3.29 \pm 0.49$ & $\ldots$ & $5.4 \mathrm{E}-03$ & 1 \\
\hline & ISOPHOT & 200 & $2.10 \pm 0.35$ & $2.03 \pm 0.34$ & $\ldots$ & $3.9 \mathrm{E}-03$ & 1 \\
\hline 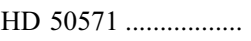 & IRAS & 60 & $0.183 \pm 0.040$ & $0.186 \pm 0.041$ & 2 & 0.011 & 4 \\
\hline 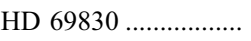 & IRAS & 25 & $0.341 \pm 0.038$ & $0.292 \pm 0.032$ & 3 & 0.142 & 4 \\
\hline HD $76582 \ldots \ldots \ldots \ldots$ & IRAS & 60 & $0.391 \pm 0.047$ & $0.403 \pm 0.048$ & 3 & $9.2 \mathrm{E}-03$ & 4 \\
\hline \multirow[t]{2}{*}{ 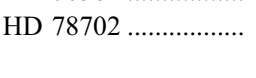 } & $I R A S$ & 60 & $0.314 \pm 0.047$ & $0.327 \pm 0.049$ & 2 & $5.6 \mathrm{E}-03$ & 4 \\
\hline & IRAS & 100 & $0.954 \pm 0.267$ & $0.987 \pm 0.276$ & 2 & $2.0 \mathrm{E}-03$ & 4 \\
\hline HD 84870A..................... & $I R A S$ & 60 & $0.243 \pm 0.046$ & $0.252 \pm 0.048$ & 3 & $2.4 \mathrm{E}-03$ & 4 \\
\hline 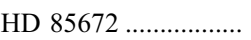 & IRAS & 60 & $0.193 \pm 0.037$ & $0.200 \pm 0.038$ & 3 & $1.4 \mathrm{E}-03$ & 4 \\
\hline \multirow[t]{2}{*}{ 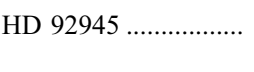 } & IRAS & 60 & $0.248 \pm 0.045$ & $0.270 \pm 0.049$ & 3 & $6.3 \mathrm{E}-03$ & 4 \\
\hline & MIPS & 70 & $0.271 \pm 0.060$ & $0.301 \pm 0.067$ & $\ldots$ & $4.6 \mathrm{E}-03$ & 7 \\
\hline 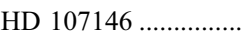 & MIPS & 24 & $0.060 \pm 0.003$ & $0.059 \pm 0.003$ & $\ldots$ & 0.042 & 2 \\
\hline & $I R A S$ & 60 & $0.705 \pm 0.056$ & $0.777 \pm 0.062$ & 3 & $6.7 \mathrm{E}-03$ & 4 \\
\hline & MIPS & 70 & $0.648 \pm 0.014$ & $0.727 \pm 0.016$ & $\ldots$ & $4.9 \mathrm{E}-03$ & 2 \\
\hline & $I R A S$ & 100 & $0.910 \pm 0.155$ & $0.913 \pm 0.155$ & 2 & $2.4 \mathrm{E}-03$ & 4 \\
\hline & SCUBA & 450 & $0.130 \pm 0.040$ & $0.130 \pm 0.040$ & $\ldots$ & $1.1 \mathrm{E}-04$ & 8 \\
\hline & ISOPHOT & 60 & $0.387 \pm 0.085$ & $0.387 \pm 0.085$ & $\ldots$ & $9.8 \mathrm{E}-04$ & 1 \\
\hline & ISOPHOT & 90 & $0.218 \pm 0.050$ & $0.201 \pm 0.046$ & $\ldots$ & $4.3 \mathrm{E}-04$ & 1 \\
\hline HD $115116 \ldots \ldots \ldots \ldots \ldots . .$. & IRAS & 60 & $0.192 \pm 0.054$ & $0.198 \pm 0.056$ & 2 & $2.5 \mathrm{E}-03$ & 4 \\
\hline 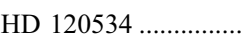 & IRAS & 60 & $0.315 \pm 0.047$ & $0.326 \pm 0.049$ & $\ldots$ & $3.3 \mathrm{E}-03$ & 4 \\
\hline 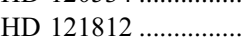 & IRAS & 60 & $0.263 \pm 0.047$ & $0.289 \pm 0.052$ & 3 & $2.7 \mathrm{E}-03$ & 4 \\
\hline & $I R A S$ & 100 & $0.484 \pm 0.135$ & $0.492 \pm 0.138$ & 2 & $9.6 \mathrm{E}-04$ & 4 \\
\hline 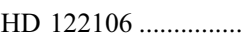 & IRAS & 60 & $0.161 \pm 0.042$ & $0.164 \pm 0.043$ & 2 & $9.2 \mathrm{E}-03$ & 4 \\
\hline 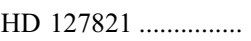 & IRAS & 60 & $0.344 \pm 0.041$ & $0.376 \pm 0.045$ & 3 & 0.011 & 4 \\
\hline & IRAS & 100 & $0.525 \pm 0.137$ & $0.529 \pm 0.138$ & 2 & $3.8 \mathrm{E}-03$ & 4 \\
\hline 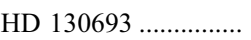 & IRAS & 60 & $0.149 \pm 0.042$ & $0.154 \pm 0.043$ & $\ldots$ & $3.2 \mathrm{E}-03$ & 4 \\
\hline 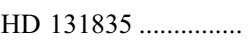 & IRAS & 25 & $0.186 \pm 0.034$ & $0.224 \pm 0.040$ & 2 & $6.1 \mathrm{E}-03$ & 4 \\
\hline & IRAS & 60 & $0.684 \pm 0.062$ & $0.681 \pm 0.061$ & 3 & $1.0 \mathrm{E}-03$ & 4 \\
\hline HD $157728 \ldots \ldots \ldots \ldots . . . .$. & IRAS & 25 & $0.213 \pm 0.019$ & $0.221 \pm 0.020$ & 3 & 0.053 & 4 \\
\hline & IRAS & 60 & $0.536 \pm 0.048$ & $0.531 \pm 0.048$ & 3 & $9.0 \mathrm{E}-03$ & 4 \\
\hline 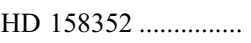 & IRAS & 25 & $0.258 \pm 0.044$ & $0.255 \pm 0.043$ & 3 & 0.075 & 4 \\
\hline & IRAS & 60 & $0.366 \pm 0.062$ & $0.348 \pm 0.059$ & 2 & 0.013 & 4 \\
\hline HD 164249 ................. & IRAS & 60 & $0.647 \pm 0.091$ & $0.669 \pm 0.094$ & 2 & $4.7 \mathrm{E}-03$ & 4 \\
\hline & ISOPHOT & 60 & $0.740 \pm 0.037$ & $0.761 \pm 0.038$ & $\ldots$ & $4.7 \mathrm{E}-03$ & 1 \\
\hline & ISOPHOT & 90 & $0.568 \pm 0.040$ & $0.560 \pm 0.039$ & $\ldots$ & $2.1 \mathrm{E}-03$ & 1 \\
\hline HD $169666 \ldots \ldots \ldots \ldots . . .$. & MIPS & 24 & $0.087 \pm 0.009$ & $0.090 \pm 0.009$ & $\ldots$ & 0.039 & 5 \\
\hline & IRAS & 25 & $0.101 \pm 0.013$ & $0.088 \pm 0.011$ & 3 & 0.036 & 4 \\
\hline 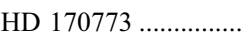 & IRAS & 60 & $0.504 \pm 0.086$ & $0.555 \pm 0.094$ & 2 & $9.1 \mathrm{E}-03$ & 4 \\
\hline & ISOPHOT & 60 & $0.547 \pm 0.027$ & $0.562 \pm 0.028$ & $\ldots$ & $9.1 \mathrm{E}-03$ & 1 \\
\hline & ISOPHOT & 90 & $0.771 \pm 0.054$ & $0.821 \pm 0.057$ & $\ldots$ & $4.0 \mathrm{E}-03$ & 1 \\
\hline 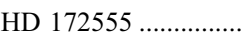 & IRAS & 12 & $1.52 \pm 0.06$ & $1.34 \pm 0.05$ & 3 & 0.508 & 4 \\
\hline & IRAS & 25 & $1.09 \pm 0.06$ & $0.91 \pm 0.05$ & 3 & 0.119 & 4 \\
\hline
\end{tabular}


TABLE 2-Continued

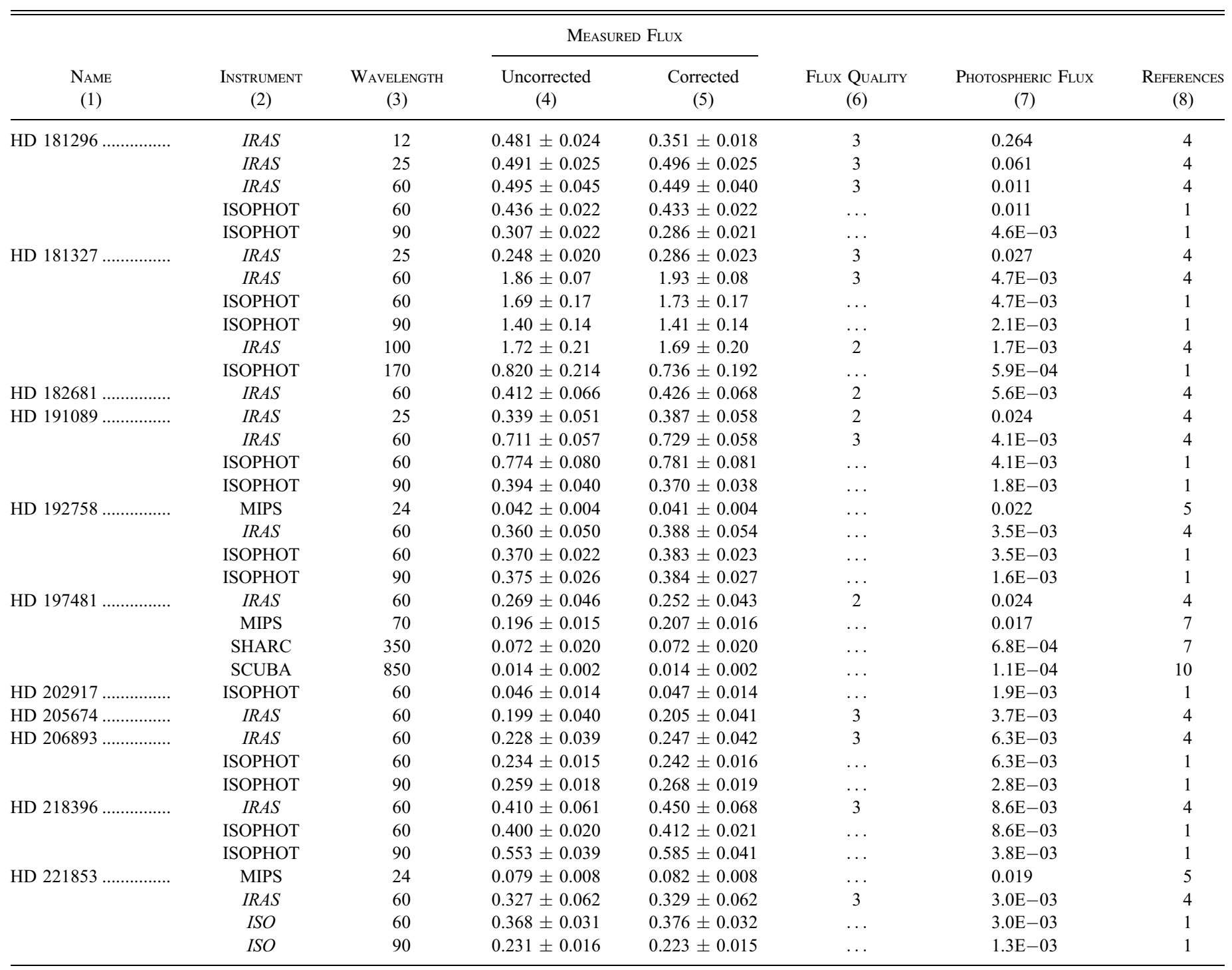

Notes.-Col. (1): Names. Col. (2): Instrument. Col. (3): Wavelength (in $\mu \mathrm{m})$. Cols. (4) and (5): Measured flux density and uncertainty (in Jy) at the specific wavelength. On the infrared flux density values in col. (5) color correction was applied. No color correction was applied on submillimeter fluxes. Col. (6): Quality of flux density, if available. Col. (7): Predicted flux density of the stellar photosphere (in Jy) at the specific wavelength. For a detailed description of the prediction method, see $\S 2.1$. Col. (8): References for papers related to the quoted flux and its uncertainty in cols. (4) and (5).

${ }^{\text {a }}$ No quoted flux uncertainty in the IRAS Serendipitous Survey Catalog. We assumed $\delta F_{25}=0.15 \mathrm{Jy}$ and $\delta F_{60}=0.32 \mathrm{Jy}$ ( $4 \%$ relative uncertainty in both cases).

REFERENCES.-(1) P. Ábrahám et al. 2006, in preparation; (2) http://data.spitzer.caltech.edu/popular/feps/20051123_enhanced_v1, FEPS Data Explanatory Supplement v3.0, Hines et al. 2005; (3) Kleinmann et al. 1986; (4) Moshir et al. 1989; (5) A. Moór et al. 2006, in preparation; (6) Rieke et al. 2005; (7) Chen et al. 2005b; (8) Williams et al. 2004; (9) Greaves et al. 2000; (10) Liu et al. 2004

Decin et al. [2000] derived significantly higher values at $60 \mu \mathrm{m}$; the probable explanation is that we used a more advanced version, ver. 10.0, of the PIA software).

As was discussed in $\S 2.1$, during the analysis we rejected several systems as bogus disks or suspicious objects. Those rejected stars that were previously proposed to harbor debris disks in the literature and would have been included in our final list (on the basis of their quoted fractional luminosity in the original paper) are presented in Appendix A, together with a brief description of the reason of rejection.

\section{AGE DETERMINATION}

\subsection{Membership in Young Moving Groups}

Age determination for main-sequence field stars is challenging and sometimes results in very uncertain values. Ages of open cluster members, however, can be estimated more accurately, e.g., by fitting their main-sequence locus in the color-magnitude diagram (CMD) with theoretical isochrones or by determining the location of the "lithium depletion edge" in the cluster and comparing it with the predictions of theoretical evolutionary models. A number of young clusters ( $\alpha$ Per, Pleiades, Hyades, etc.) have been dated so far (Meynet et al. 1993; Stauffer et al. 1998). Similarly, the ages of young stellar kinematic groups, discovered mainly in recent years, are relatively well determined (e.g., Zuckerman \& Song 2004b). It was a very important result that several stars with the strongest infrared excess turned out to be members of such moving groups, and in some cases the ages of these stars had to be revised significantly (e.g., the case of $\beta$ Pic; Barrado y Navascués et al. 1999). In order to obtain more reliable ages for our sample, we performed a systematic investigation of the possible relationship between our excess stars and 
nearby kinematic moving groups, stellar associations, or open clusters.

A common method to decide whether an object belongs to a moving group is to compare its Galactic space velocity components with the mean velocity components of the group. In order to compute the space velocity for the stars in Table 1, we collected parallaxes and proper motions from the Hipparcos and Tycho-2 catalogs. When accurate parallax information was not available, a photometric distance was adopted.

Radial velocities were taken from the literature (see Table 3 for references) or from our own observations. The new observations were carried out with the $2.3 \mathrm{~m}$ ANU telescope at the Siding Spring Observatory, Australia, on 11 nights between 2005 March 21 and August 22. The spectra were taken with the Double Beam Spectrograph using a $1200 \mathrm{~mm}^{-1}$ grating in the red arm. The recorded spectra covered $1000 \AA$ A between 5800 and $6800 \AA$, with a dispersion of $0.55 \AA$ pixel $^{-1}$. This leads to a nominal resolution of about $1 \AA$. The exposure time ranged between 30 and $200 \mathrm{~s}$ depending on the brightness of the target and the weather conditions. We obtained on average four to seven spectra for each star. Since all of the target stars are bright objects $(V<10$ mag), we could easily reach $\mathrm{S} / \mathrm{N} \sim 150-200$ for every spectrum. All spectra were reduced with standard tasks in IRAF. ${ }^{10}$ Reduction consisted of bias and flat-field corrections, aperture extraction, wavelength calibration, and continuum normalization. We did not attempt flux calibration because the conditions were often nonphotometric and the main aim was to measure radial velocities. Radial velocities were determined by cross-correlation, using the IRAF task fxcor, choosing HD 187691 as a stable IAU velocity standard. The cross-correlated region was $100 \AA$ centered on the $\mathrm{H} \alpha$ line, which is by far the strongest spectral feature in our range. The finally adopted velocities were calculated as simple mean values of the individual measurements. Our experiences have shown that the typical measurement errors were about $4-7 \mathrm{~km} \mathrm{~s}^{-1}$ per point, so that the mean values have $\pm 1-3 \mathrm{~km} \mathrm{~s}^{-1}$ standard deviations. These were adopted as the uncertainties shown in Table 3.

In the calculation of the Galactic space velocity we used a right-handed coordinate system ( $U$ is positive toward the Galactic center, $V$ is positive in the direction of Galactic rotation, and $W$ is positive toward the north Galactic pole) and followed the general recipe described in the Hipparcos and Tycho catalogs (ESA 1997). The computed Galactic space velocity components and their uncertainties are given in Table 3.

Table 4 summarizes the basic properties of the relevant moving groups and associations within $120 \mathrm{pc}$ from the Sun. The probability that star $i$ is a member of moving group $j$ can be computed by

$$
\begin{aligned}
P_{i j}=\exp \left\{-\left[\frac{\left(U_{i}-U_{j}\right)^{2}}{2\left(\sigma_{U_{i}}^{2}+\sigma_{U_{j}}^{2}\right)}+\frac{\left(V_{i}-V_{j}\right)^{2}}{2\left(\sigma_{V_{i}}^{2}+\sigma_{V_{j}}^{2}\right)}\right.\right. \\
\left.\left.+\frac{\left(W_{i}-W_{j}\right)^{2}}{2\left(\sigma_{W_{i}}^{2}+\sigma_{W_{j}}^{2}\right)}\right]\right\},
\end{aligned}
$$

where $U_{i}, V_{i}, W_{i}$ and $\sigma_{U_{i}}, \sigma_{V_{i}}, \sigma_{W_{i}}$ are the Galactic space velocity components of the star and their uncertainties, respectively, while $U_{j}, V_{j}, W_{j}$ and $\sigma_{U_{j}}, \sigma_{V_{j}}, \sigma_{W_{j}}$ are the mean Galactic space velocity components of the specific kinematic group and the cor-

\footnotetext{
10 IRAF is distributed by the National Optical Astronomy Observatory, which is operated by the Association of Universities for Research in Astronomy, Inc., under cooperative agreement with the National Science Foundation.
}

responding errors. In this formula we assumed that the velocity distribution within a group is Gaussian. When $\sigma_{U_{j}}, \sigma_{V_{j}}, \sigma_{W_{j}}$ parameters were not available in the literature, we computed them from the velocity dispersion of known members around the mean. Our newly calculated mean values were always consistent with those from the literature within the uncertainties. In those few cases where no sufficient membership information could be found in the literature, we adopted $\sigma_{U_{j}}=\sigma_{V_{j}}=\sigma_{W_{j}}=2 \mathrm{~km} \mathrm{~s}^{-1}$ (a characteristic value in the previous cases).

Probability values for each star with respect to each group were computed. Then we checked the resulting $P_{i j}$ values for objects already assigned to a group in the literature. The numbers spread in the range of $0.2<P<1.0$; therefore, we set $P=0.2$ as a lower limit for the new moving group member candidates as well. Stars assigned to any group above this threshold were further checked by comparing their three-dimensional space location with the volume occupied by the group (most groups are rather confined in space). There were a few stars that could be assigned to both the Tucana-Horologium and GAYA2 associations; these cases are analyzed in Appendix B. Table 3 presents the final assignments between stars and kinematic groups.

From our sample of 60 objects, 26 sources could be linked to stellar associations; 13 of them are new members identified in the present study. For 10 stars out of these 13, age estimates are available in the literature. For these 10 objects we directly compare our age estimates with previous values in Table 5. In most cases ages derived in our study are younger than the earlier values. The discrepancy is particularly obvious in the case of ages derived from isochrone fitting (e.g., Nordström et al. 2004). However, this problem is not related only to the present study. For example, HD 105 has a moving group age of $30_{-20}^{+10} \mathrm{Myr}$ (Mamajek et al. 2004), while Nordström et al. (2004) quoted $8600_{-3800}^{+4000} \mathrm{Myr}$ from isochrone fitting. Similar objects in our sample are HD 25457 (50-100 Myr from moving group, Zuckerman \& Song 2004b; $4000_{-2100}^{+1200}$ Myr from isochrones, Nordström et al. 2004), HD $164249\left(12_{-4}^{+8} \mathrm{Myr}\right.$, Song et al. 2003; 2200 ${ }_{-1800}^{+1200} \mathrm{Myr}$, Nordström et al. 2004), and HD 181327 (12 $2_{-4}^{+8}$ Myr, Song et al. 2003; $1300_{-1300}^{+1000}$ Myr, Nordström et al. 2004). The age uncertainty related to isochrone fitting might arise from the lack of information on whether the star is in the pre-main-sequence phase of its evolution or is an evolved object above the main sequence. In ambiguous cases we always adopted age estimates derived from stellar kinematic group membership.

\subsection{Statistical Age Estimates for the Disk Sample}

For stars not assigned to any moving groups, other age estimation methods are needed. Before focusing on individual systems, in this subsection we analyze what can be learned about the age distribution of our sample of debris disk systems.

\subsubsection{Distribution of the Excess Stars in the Velocity Space}

The distribution of the derived Galactic space velocities (Table 3 ) is displayed in Figures $1 a$ and $1 b$. Overplotted is the box occupied by young disk population stars defined by Leggett (1992) on the basis of a systematic study of Eggen (1989). The plots show that most stars from our sample belong to this population. This fact suggests that the majority of our sample of stars from Table 1 are relatively young.

\subsubsection{Location of A-Type Stars on the CMD}

For a sample of bright A-type stars Jura et al. (1998) demonstrated that three objects with strong infrared excess (HR 4796A, HD 9672, $\beta$ Pic) are located close to the lower boundary of the distribution in the CMD. Comparing their locations with the loci 
TABLE 3

Radial Velocity Information and Membership in Moving Groups

\begin{tabular}{|c|c|c|c|c|c|c|c|c|}
\hline $\begin{array}{l}\text { Name } \\
(1)\end{array}$ & $\begin{array}{c}V_{\mathrm{rad}} \\
\left(\mathrm{km} \mathrm{s}^{-1}\right) \\
(2)\end{array}$ & $\begin{array}{c}\text { References } \\
\text { (3) }\end{array}$ & $\begin{array}{c}U \\
\left(\mathrm{~km} \mathrm{~s}^{-1}\right) \\
(4)\end{array}$ & $\begin{array}{c}V \\
\left(\mathrm{~km} \mathrm{~s}^{-1}\right) \\
(5)\end{array}$ & $\begin{array}{c}W \\
\left(\mathrm{~km} \mathrm{~s}^{-1}\right) \\
(6)\end{array}$ & $\begin{array}{c}\text { Association SKG } \\
\text { (7) }\end{array}$ & $\begin{array}{c}\text { Probability } \\
(\%) \\
(8)\end{array}$ & $\begin{array}{c}\text { References } \\
\text { (9) }\end{array}$ \\
\hline 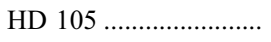 & $+1.6 \pm 0.3$ & 1 & $-9.8 \pm 0.4$ & $-21.5 \pm 0.8$ & $-1.3 \pm 0.3$ & TucHor & 78 & 10 \\
\hline 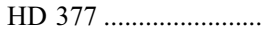 & $+1.3 \pm 0.3$ & 1 & $-14.4 \pm 0.6$ & $-7.0 \pm 0.4$ & $-3.9 \pm 0.3$ & $\ldots$ & $\ldots$ & 11 \\
\hline 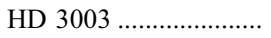 & $+7.0 \pm 2.0$ & 2 & $-9.6 \pm 0.8$ & $-20.9 \pm 1.0$ & $-0.6 \pm 1.6$ & TucHor & 85 & 11 \\
\hline 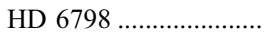 & $+10.0 \pm 4.3$ & 3 & $-36.6 \pm 2.7$ & $-13.5 \pm 3.5$ & $+4.1 \pm 1.3$ & $\ldots$ & $\ldots$ & $\ldots$ \\
\hline 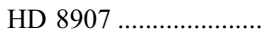 & $+8.8 \pm 0.4$ & 1 & $-10.4 \pm 0.3$ & $-4.6 \pm 0.4$ & $-16.7 \pm 0.4$ & $\ldots$ & $\ldots$ & $\ldots$ \\
\hline 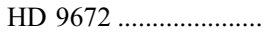 & $+11.4 \pm 1.8$ & 3 & $-23.9 \pm 1.1$ & $-16.6 \pm 0.8$ & $-6.6 \pm 1.7$ & $\ldots$ & $\ldots$ & $\ldots$ \\
\hline 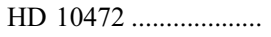 & $+19.5 \pm 1.6$ & 4 & $-8.5 \pm 0.7$ & $-24.2 \pm 1.1$ & $-10.4 \pm 1.3$ & LA & 38 & 4 \\
\hline 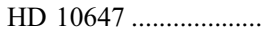 & $+27.5 \pm 0.2$ & 1 & $-1.2 \pm 0.1$ & $-26.6 \pm 0.2$ & $-17.8 \pm 0.2$ & $\ldots$ & $\ldots$ & $\ldots$ \\
\hline HD $10638 \ldots \ldots \ldots \ldots \ldots . . . . . . . .$. & $-0.4 \pm 1.2$ & 5 & $-12.3 \pm 1.0$ & $-25.0 \pm 1.5$ & $-14.8 \pm 1.0$ & LA & 29 & 4 \\
\hline 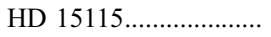 & $+8.8 \pm 3.0$ & 4 & $-13.2 \pm 1.9$ & $-17.8 \pm 1.2$ & $-6.0 \pm 2.3$ & BPMG & 22 & 4 \\
\hline HD $15745 \ldots \ldots \ldots \ldots \ldots . . . . . . .$. & $+10.5 \pm 1.2$ & 4 & $-16.5 \pm 1.1$ & $-10.8 \pm 1.3$ & $-10.7 \pm 0.7$ & $\ldots$ & $\ldots$ & $\ldots$ \\
\hline 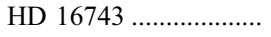 & $+21.9 \pm 1.1$ & 4 & $-23.6 \pm 0.9$ & $-18.0 \pm 0.6$ & $-15.1 \pm 0.9$ & $\ldots$ & $\ldots$ & $\ldots$ \\
\hline 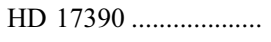 & $+7.2 \pm 1.8$ & 1 & $-15.3 \pm 0.8$ & $-9.6 \pm 0.5$ & $+1.0 \pm 1.6$ & $\ldots$ & $\ldots$ & $\ldots$ \\
\hline 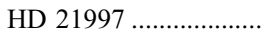 & $+17.3 \pm 0.8$ & 3 & $-12.9 \pm 0.5$ & $-22.3 \pm 1.0$ & $-3.9 \pm 0.9$ & GAYA2 & 62 & 4 \\
\hline 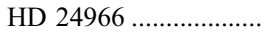 & $+29.6 \pm 2.2$ & 4 & $-15.0 \pm 0.9$ & $-26.4 \pm 1.4$ & $-13.2 \pm 1.8$ & $\ldots$ & $\ldots$ & $\ldots$ \\
\hline 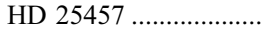 & $+17.6 \pm 0.2$ & 1 & $-7.9 \pm 0.2$ & $-28.7 \pm 0.4$ & $-11.9 \pm 0.2$ & AB Dor & 30 & 12 \\
\hline 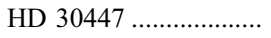 & $+21.3 \pm 2.5$ & 4 & $-13.1 \pm 1.4$ & $-20.9 \pm 1.6$ & $-3.8 \pm 1.7$ & GAYA2 & 54 & 4 \\
\hline 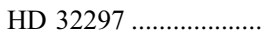 & $+21.8 \pm 2.1$ & 4 & $-16.3 \pm 2.0$ & $-16.0 \pm 1.5$ & $-11.0 \pm 1.0$ & $\ldots$ & $\cdots$ & $\cdots$ \\
\hline 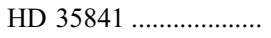 & $+23.1 \pm 1.3$ & 4 & $-13.0 \pm 1.0$ & $-21.3 \pm 1.7$ & $-3.6 \pm 1.7$ & GAYA2 & 56 & 4 \\
\hline 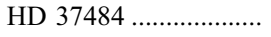 & $+23.0 \pm 2.6$ & 4 & $-11.6 \pm 1.4$ & $-20.4 \pm 1.9$ & $-5.2 \pm 1.3$ & TucHor & 25 & 4 \\
\hline 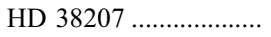 & $+24.9 \pm 1.4$ & 4 & $-14.7 \pm 1.1$ & $-21.2 \pm 1.5$ & $-4.1 \pm 1.5$ & GAYA2 & 32 & 4 \\
\hline 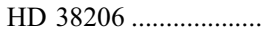 & $+24.9 \pm 0.6$ & 3 & $-13.7 \pm 0.5$ & $-21.2 \pm 0.5$ & $-6.1 \pm 0.4$ & GAYA2 & 27 & 4 \\
\hline 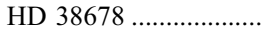 & $+18.9 \pm 2.7$ & 3 & $-13.6 \pm 2.0$ & $-10.5 \pm 1.6$ & $-8.1 \pm 1.0$ & Castor & 28 & 13 \\
\hline 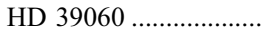 & $+20.2 \pm 0.4$ & 6 & $-10.9 \pm 0.1$ & $-16.2 \pm 0.3$ & $-9.2 \pm 0.2$ & BPMG & 98 & 14 \\
\hline 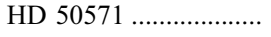 & $+22.2 \pm 1.2$ & 1 & $-16.2 \pm 0.4$ & $-22.3 \pm 1.1$ & $-4.4 \pm 0.5$ & $\ldots$ & $\ldots$ & $\ldots$ \\
\hline 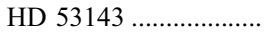 & $+21.9 \pm 0.1$ & 1 & $-25.5 \pm 0.3$ & $-18.1 \pm 0.1$ & $-15.2 \pm 0.1$ & $\ldots$ & $\ldots$ & $\ldots$ \\
\hline 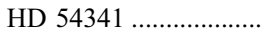 & $+47.4 \pm 1.4$ & 4 & $-16.5 \pm 0.5$ & $-44.0 \pm 1.3$ & $-8.7 \pm 0.5$ & $\ldots$ & $\ldots$ & $\ldots$ \\
\hline 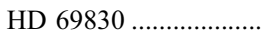 & $+29.8 \pm 0.1$ & 1 & $+28.9 \pm 0.5$ & $-60.9 \pm 0.4$ & $-10.1 \pm 0.2$ & $\cdots$ & $\cdots$ & $\cdots$ \\
\hline HD $76582 \ldots \ldots \ldots \ldots \ldots . . . . . . . .$. & $-4.0 \pm 2.5$ & 5 & $+10.7 \pm 1.8$ & $+5.1 \pm 1.1$ & $+9.9 \pm 1.5$ & $\ldots$ & $\ldots$ & $\ldots$ \\
\hline 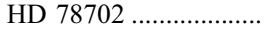 & $+16.9 \pm 2.1$ & 3 & $-27.5 \pm 1.6$ & $-10.5 \pm 1.8$ & $-7.3 \pm 1.1$ & $\ldots$ & $\cdots$ & $\cdots$ \\
\hline HD 84870A..................... & $+3.7 \pm 0.4$ & 4 & $-7.8 \pm 0.5$ & $-25.5 \pm 2.0$ & $-5.7 \pm 0.7$ & $\ldots$ & $\ldots$ & $\ldots$ \\
\hline HD $85672 \ldots \ldots \ldots \ldots \ldots . . . . . . . .$. & $-5.2 \pm 6.8$ & 7 & $-9.5 \pm 4.2$ & $-3.0 \pm 1.6$ & $-14.8 \pm 5.4$ & $\ldots$ & $\cdots$ & $\ldots$ \\
\hline 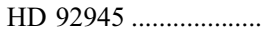 & $+22.6 \pm 0.2$ & 1 & $-15.2 \pm 0.3$ & $-27.8 \pm 0.2$ & $-4.3 \pm 0.3$ & $\cdots$ & $\cdots$ & $\ldots$ \\
\hline 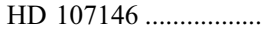 & $+1.5 \pm 0.2$ & 1 & $-10.6 \pm 0.3$ & $-28.8 \pm 0.7$ & $-5.2 \pm 0.3$ & $\ldots$ & $\ldots$ & $\ldots$ \\
\hline HD 109573A.................. & $+9.4 \pm 2.3$ & 3 & $-9.0 \pm 1.3$ & $-19.0 \pm 1.9$ & $-4.4 \pm 1.0$ & TWA & 64 & 15 \\
\hline HD $110058 \ldots \ldots \ldots \ldots \ldots . . . . . . .$. & $+21.7 \pm 1.3$ & 4 & $+0.0 \pm 1.3$ & $-26.6 \pm 1.4$ & $-2.1 \pm 0.8$ & $\ldots$ & $\ldots$ & $\ldots$ \\
\hline 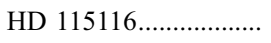 & $-2.3 \pm 0.7$ & 7 & $-30.5 \pm 1.5$ & $-17.2 \pm 1.1$ & $-1.8 \pm 0.3$ & $\cdots$ & $\cdots$ & $\cdots$ \\
\hline HD $120534 \ldots \ldots \ldots \ldots \ldots . . . . . . . .$. & $+46.7 \pm 1.1$ & 4 & $+21.8 \pm 1.8$ & $-43.1 \pm 3.2$ & $+15.0 \pm 1.8$ & $\ldots$ & $\ldots$ & $\ldots$ \\
\hline 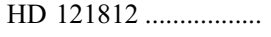 & $-15.2 \pm 0.7$ & 8 & $-29.6 \pm 1.2$ & $-61.3 \pm 2.7$ & $-1.8 \pm 0.9$ & $\ldots$ & $\cdots$ & $\cdots$ \\
\hline HD $122106 \ldots \ldots \ldots \ldots \ldots . . . . . . .$. & $-1.6 \pm 5.0$ & 1 & $+0.6 \pm 2.6$ & $-21.7 \pm 2.0$ & $-9.0 \pm 4.2$ & $\ldots$ & $\ldots$ & $\ldots$ \\
\hline 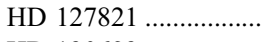 & $-15.4 \pm 2.6$ & 1 & $-16.0 \pm 0.5$ & $-26.2 \pm 1.6$ & $-2.7 \pm 2.0$ & $\cdots$ & $\cdots$ & $\ldots$ \\
\hline HD $130693 \ldots \ldots \ldots . . . . . . .$. & $+14.3 \pm 0.8$ & 4 & $+8.1 \pm 0.9$ & $-7.3 \pm 0.6$ & $+10.4 \pm 0.8$ & $\ldots$ & $\ldots$ & $\ldots$ \\
\hline HD $131835 \ldots \ldots \ldots \ldots \ldots . . . . . . . .$. & $+3.3 \pm 1.7$ & 4 & $-4.6 \pm 1.6$ & $-17.2 \pm 2.1$ & $-3.0 \pm 1.0$ & UCL & 50 & 16 \\
\hline HD $157728 \ldots \ldots \ldots . . . . . . . . .$. & $-19.7 \pm 1.2$ & 5 & $-7.0 \pm 0.8$ & $-21.3 \pm 0.8$ & $-4.4 \pm 0.6$ & $\ldots$ & $\ldots$ & $\ldots$ \\
\hline HD $158352 \ldots \ldots \ldots \ldots \ldots . . . . . . .$. & $-36.1 \pm 1.2$ & 5 & $-35.5 \pm 1.1$ & $-19.9 \pm 0.5$ & $+7.4 \pm 0.9$ & $\ldots$ & $\ldots$ & $\ldots$ \\
\hline HD 164249 ..................... & $-0.2 \pm 0.5$ & 1 & $-7.6 \pm 0.6$ & $-15.3 \pm 0.7$ & $-8.9 \pm 0.4$ & BPMG & 20 & 17 \\
\hline HD $169666 \ldots \ldots \ldots \ldots . . . . . . .$. & $-44.3 \pm 0.6$ & 1 & $+1.3 \pm 0.3$ & $-46.8 \pm 0.6$ & $-7.9 \pm 0.5$ & $\ldots$ & $\ldots$ & $\ldots$ \\
\hline HD 170773 ...................... & $-26.3 \pm 1.1$ & 1 & $-30.5 \pm 1.1$ & $-3.8 \pm 0.2$ & $-12.3 \pm 0.6$ & $\ldots$ & $\cdots$ & $\cdots$ \\
\hline 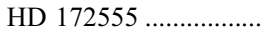 & $+2.0 \pm 2.5$ & 5 & $-11.0 \pm 2.0$ & $-15.6 \pm 1.2$ & $-9.3 \pm 1.0$ & BPMG & 96 & 17 \\
\hline 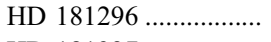 & $-2.0 \pm 10.0$ & 2 & $-10.7 \pm 8.6$ & $-14.9 \pm 2.7$ & $-7.3 \pm 4.4$ & BPMG & 87 & 17 \\
\hline HD 181327 .................... & $+0.2 \pm 0.4$ & 1 & $-9.1 \pm 0.5$ & $-16.2 \pm 0.7$ & $-8.4 \pm 0.4$ & BPMG & 57 & 17 \\
\hline 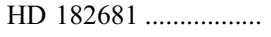 & $+1.4 \pm 5.0$ & 9 & $-0.4 \pm 4.6$ & $-13.4 \pm 1.1$ & $-10.8 \pm 1.8$ & $\ldots$ & $\ldots$ & $\ldots$ \\
\hline HD 191089 ..................... & $-5.8 \pm 2.2$ & 1 & $-7.8 \pm 1.9$ & $-16.2 \pm 0.9$ & $-10.3 \pm 1.2$ & BPMG & 38 & 4 \\
\hline HD $192758 \ldots \ldots \ldots \ldots . . . . . .$. & $-11.1 \pm 1.2$ & 4 & $-18.4 \pm 2.1$ & $-13.8 \pm 2.8$ & $-6.7 \pm 2.7$ & IC 2391 & 50 & 4 \\
\hline HD $197481 \ldots \ldots \ldots \ldots . . . . . . . .$. & $-4.5 \pm 1.3$ & 3 & $-10.1 \pm 1.0$ & $-16.4 \pm 0.3$ & $-10.5 \pm 0.8$ & BPMG & 56 & 14 \\
\hline 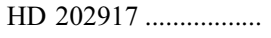 & $-1.6 \pm 0.2$ & 1 & $-8.2 \pm 0.4$ & $-20.0 \pm 1.1$ & $-0.3 \pm 0.2$ & TucHor & 48 & 11 \\
\hline 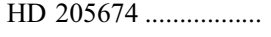 & $+1.1 \pm 5.1$ & 4 & $-1.6 \pm 3.0$ & $-24.5 \pm 2.4$ & $-16.6 \pm 3.7$ & $\ldots$ & $\cdots$ & $\cdots$ \\
\hline HD $206893 \ldots \ldots \ldots \ldots . . . . . .$. & $-12.9 \pm 1.4$ & 1 & $-20.0 \pm 0.9$ & $-7.7 \pm 0.7$ & $-2.1 \pm 1.0$ & $\ldots$ & $\ldots$ & $\ldots$ \\
\hline 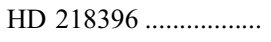 & $-12.6 \pm 1.3$ & 3 & $-12.4 \pm 0.5$ & $-21.4 \pm 1.1$ & $-7.4 \pm 0.9$ & LA & 62 & 4 \\
\hline HD $221853 \ldots \ldots \ldots \ldots . . . . . . . .$. & $-4.2 \pm 2.1$ & 4 & $-12.7 \pm 0.9$ & $-20.6 \pm 1.8$ & $-11.2 \pm 1.9$ & LA & 94 & 4 \\
\hline
\end{tabular}

Notes.-Col. (1): Names. Col. (2): Radial velocity and its uncertainty. Col. (3): References for the source of measurement. Cols. (4)-(6): $U$, $V$, $W$ Galactic space

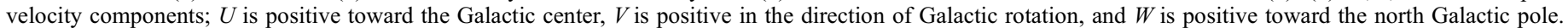
Col. (7): Assigned stellar kinematic group (see Table 4). Col. (8): Membership probability. Col. (9): References for membership identification.

REFERENCES.- (1) Nordström et al. 2004 and references therein; (2) Zuckerman \& Webb 2000; (3) Kharchenko et al. 2004 and references therein; (4) this paper; (5) Wilson

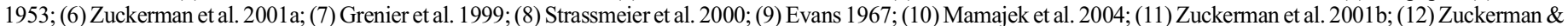
Song 2004b; (13) Barrado y Navascués 1998; (14) Barrado y Navascués et al. 1999; (15) Webb et al. 1999; (16) de Zeeuw et al. 1999; (17) Song et al. 2003. 
TABLE 4

Description of Stellar Kinematic Groups

\begin{tabular}{|c|c|c|c|c|}
\hline $\begin{array}{l}\text { Group Name } \\
\text { (1) }\end{array}$ & $\begin{array}{c}U, V, W \\
\left(\mathrm{~km} \mathrm{~s}^{-1}\right) \\
(2)\end{array}$ & $\begin{array}{c}\text { References } \\
\text { (3) }\end{array}$ & $\begin{array}{l}\text { Age } \\
\text { (Myr) } \\
(4)\end{array}$ & $\begin{array}{c}\text { References } \\
\text { (5) }\end{array}$ \\
\hline 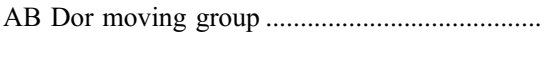 & $\begin{array}{c}-8,-27,-14 \\
\ldots\end{array}$ & $\begin{array}{c}1 \\
\ldots\end{array}$ & $\begin{array}{c}50 \\
100\end{array}$ & $\begin{array}{l}1 \\
6\end{array}$ \\
\hline$\beta$ Pictoris moving group & $-11,-16,-9$ & 1 & $12_{-4}^{+8}$ & 7 \\
\hline Castor moving group & $-10.7,-8.0,-9.7$ & 2 & $200 \pm 100$ & 2 \\
\hline 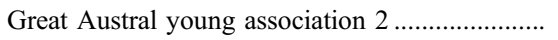 & $-11.0,-22.5,-4.6$ & 3 & 20 & 3 \\
\hline Hyades & $-40,-17,-3$ & 1 & 600 & 1 \\
\hline 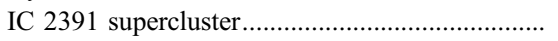 & $-20.6,-15.7,-9.1$ & 4 & $35-55$ & 4 \\
\hline 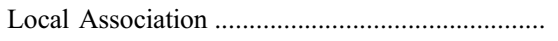 & $-11.6,-21.0,-11.4$ & 4 & $20-150$ & 4 \\
\hline 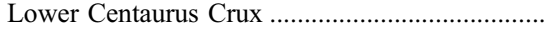 & $-8.2,-18.6,-6.4$ & 5 & $16 \pm 1$ & 8 \\
\hline Upper Centaurus Lupus............................................. & $-6.8,-19.3,-5.7$ & 5 & $17 \pm 1$ & 8 \\
\hline \multirow[t]{4}{*}{ 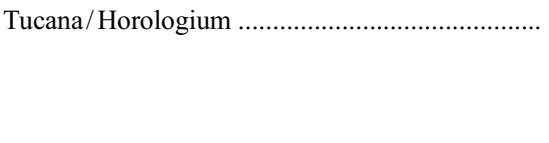 } & $-11,-21,0$ & 1 & 30 & 1 \\
\hline & $\ldots$ & $\ldots$ & $10-30$ & 9 \\
\hline & $\ldots$ & $\ldots$ & 20 & 10 \\
\hline & $\ldots$ & $\ldots$ & $10-40$ & 11 \\
\hline \multirow[t]{2}{*}{ 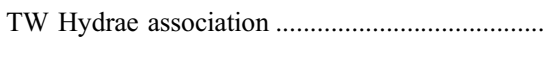 } & $-11,-18,-5$ & 1 & 8 & 1 \\
\hline & & & $5-15$ & 12 \\
\hline \multirow[t]{2}{*}{ 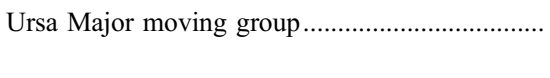 } & $+14,+1,-9$ & 1 & 300 & 1 \\
\hline & $\ldots$ & $\ldots$ & 500 & 13 \\
\hline
\end{tabular}

REFERENCES.-(1) Zuckerman \& Song 2004b and references therein; (2) Barrado y Navascués 1998; (3) Torres et al. 2003; (4) Montes et al. 2001 and references therein; (5) Sartori et al. 2003; (6) Luhman et al. 2005; (7) Zuckerman et al. 2001a; (8) Mamajek et al. 2002; (9) Stelzer \& Neuhäuser 2000; (10) Torres et al. 2000; (11) Zuckerman et al. 2001b; (12) Weintraub et al. 2000; (13) King et al. 2003.

TABLE 5

Comparison of Age Estimates for Newly Identified Moving Group Members

\begin{tabular}{|c|c|c|}
\hline Name & $\begin{array}{l}\text { Age Estimates } \\
\text { (Myr) }\end{array}$ & References \\
\hline \multirow[t]{3}{*}{ HD 10472 . } & $2000_{-1400}^{+1000}$ & 1 \\
\hline & 30 & 2 \\
\hline & {$[20,150]$} & 3 \\
\hline \multirow[t]{3}{*}{ HD 15115 } & $900_{-900}^{+1300}$ & 1 \\
\hline & 100 & 2 \\
\hline & $12_{-4}^{+8}$ & 3 \\
\hline \multirow[t]{2}{*}{ 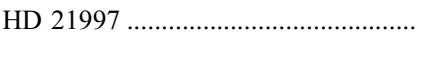 } & 100 & 2 \\
\hline & $20_{-10}^{+10}$ & 3 \\
\hline \multirow[t]{3}{*}{ 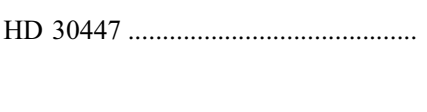 } & $2100_{-1100}^{+700}$ & 1 \\
\hline & $\lesssim 100$ & 2 \\
\hline & $20_{-10}^{+10}$ & 3 \\
\hline \multirow[t]{3}{*}{ 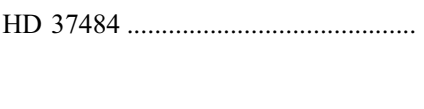 } & $1500_{-1500}^{+900}$ & 1 \\
\hline & {$[30,100]$} & 4 \\
\hline & $30_{-20}^{+10}$ & 3 \\
\hline \multirow[t]{2}{*}{ HD 38207 .......................................... } & {$[100,300]$} & 4 \\
\hline & $20_{-10}^{+10}$ & 3 \\
\hline \multirow[t]{2}{*}{ HD 38206} & $\sim 9_{-9}^{+10}$ & 5 \\
\hline & $20_{-10}^{+10}$ & 3 \\
\hline \multirow[t]{4}{*}{ HD 191089} & $17_{-4}^{+8}$ & 6 \\
\hline & $3000_{-900}^{+700}$ & 1 \\
\hline & $\lesssim 100$ & 2 \\
\hline & $12_{-4}^{+8}$ & 3 \\
\hline \multirow[t]{3}{*}{ HD $218396 \ldots \ldots \ldots$} & $732_{-682}^{-396}$ & 7 \\
\hline & 30 & 2 \\
\hline & {$[20,150]$} & 3 \\
\hline \multirow{5}{*}{ HD 221853} & 800 & 8 \\
\hline & 1800 & 9 \\
\hline & $1700_{-600}^{+500}$ & 1 \\
\hline & $\lesssim 100$ & 2 \\
\hline & {$[20,150]$} & 3 \\
\hline
\end{tabular}

REFERENCES.—(1) Nordström et al. 2004; (2) Zuckerman \& Song 2004a; (3) this paper; (4) Carpenter et al. 2005; (5) Gerbaldi et al. 1999; (6) Mamajek 2004; (7) Song et al. 2001; (8) Silverstone 2000; (9) Decin et al. 2000. of the youngest, nearby open clusters ( $\alpha$ Per, IC 2391, Pleiades), Lowrance et al. (2000) argued that stars with strong infrared excess are typically younger than these clusters. Adopting this idea, we selected stars with $B-V$ ranging between -0.1 and 0.33 (corresponding mainly to A-type stars) from Table 1 and plotted them in the CMD of Figure 2. In addition, we overplotted a volume-limited sample of A-type stars $(d<100 \mathrm{pc})$ extracted from the Hipparcos catalog (it was requested that the parallax error was less than $10 \%$ and the $B-V$ uncertainty was lower than $0.01 \mathrm{mag})$. For comparison, members of the open clusters $\alpha$ Per (80 Myr) and Hyades (600 Myr) are marked. The older cluster, Hyades, covers the upper part of the distribution, while $\alpha$ Per stars are situated at lower absolute magnitudes for the same color. Pleiades (100 Myr, not plotted in the figure for clarity) occupies the same region as $\alpha$ Per (see Fig. 3 of Lowrance et al. 2000).

Figure 2 shows that the majority of the objects from our sample of high- $f_{d}$ stars appear to be close to the lower boundary of the area occupied by A stars and are located below the region of $\alpha$ Per and Pleiades, with some overlap. This suggests that the early spectral type stars from our sample of high- $f_{d}$ disks are young, probably close to the zero-age main sequence (ZAMS), and they are presumably not older than $100 \mathrm{Myr}$, the age of the Pleiades.

\subsection{Ages of Individual Objects}

For those objects that could be assigned to one of the moving groups or associations, the age of that group, as well as its uncertainty, was adopted (26 objects). For a number of stars not associated with groups or with associations, age estimates could be found in the literature (19 stars; for references see Table 1). Sometimes literature data for a specific star scatter significantly; in these cases we adopted an age range that covers all quoted values and their uncertainties. When the literature search did not yield any dating, we made age estimates by plotting the stars on the H-R diagram and comparing their positions to isochrones. For stars 

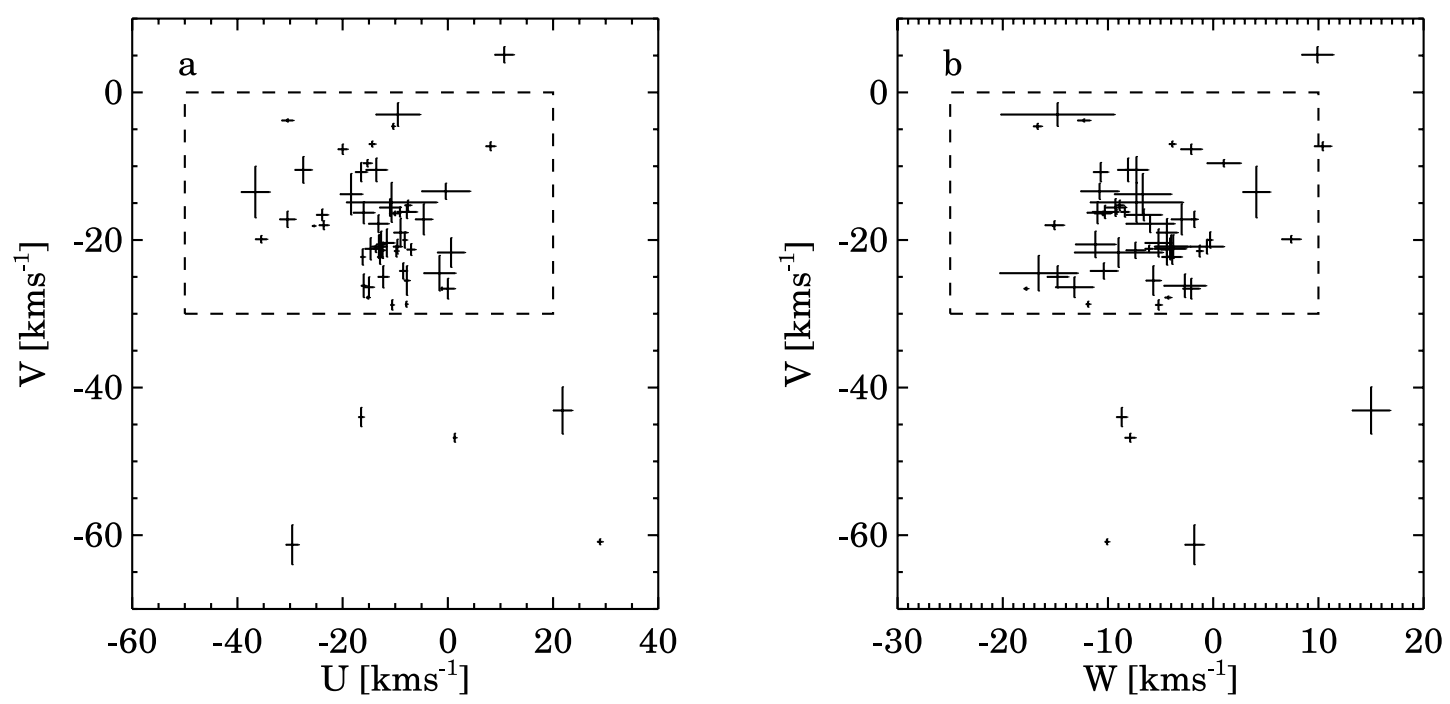

Fig. 1.- $(U, V)$ - and $(W, V)$-planes for stars in Table 3. The dashed rectangle marks the young disk population as defined by Leggett (1992).

with spectral types in the range B9-G5 the isochrone age was estimated following the general outline described by Lachaume et al. (1999), using the Padova theoretical isochrones (Girardi et al. 2000). This method was applied to 13 objects.

This isochrone method gives only upper limits for some A-type stars. As a best estimate for these stars (five cases), we adopted an upper limit of $100 \mathrm{Myr}$, consistent with our results shown in Figure 2 and discussed in $\S$ 3.2.2. For stars of later spectral type there are some widely used age indicators, like the strength of the $\mathrm{Ca}$ II $\mathrm{H}$ and $\mathrm{K}$ lines or the X-ray luminosity of the star. In the case of HD 121812 our age estimate is based on the former method, taking the measured value from Strassmeier et al. (2000) and using the calibration of Lachaume et al. (1999). HD 130693 has a ROSAT counterpart and its X-ray luminosity of $\log L_{\mathrm{X}}=29.7 \mathrm{ergs} \mathrm{s}^{-1}$ was compared with the X-ray luminosity distribution function of late-type members in different

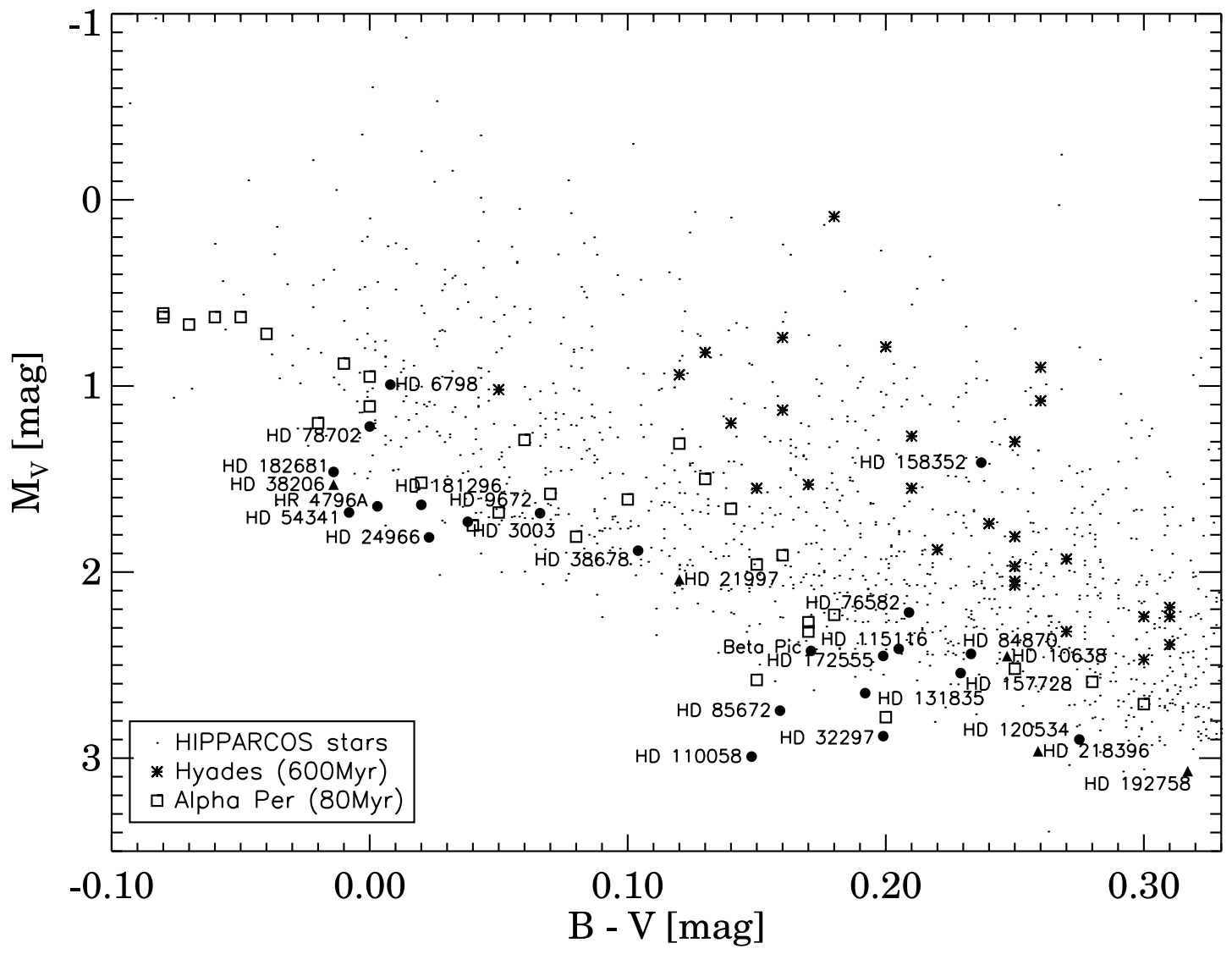

Fig. 2.-CMD of A-type stars. Objects measured by Hipparcos within $100 \mathrm{pc}$ from the Sun with parallax error $(<10 \%)$ and $(B-V)$ error $(<0.01 \mathrm{mag})$ are represented by small dots. Filled symbols mark positions of A- and F0-type stars from Table 1; triangles correspond to objects assigned to any stellar kinematic group in this work. Stars of $\alpha$ Per and Hyades open clusters are represented by squares and asterisks, respectively. 


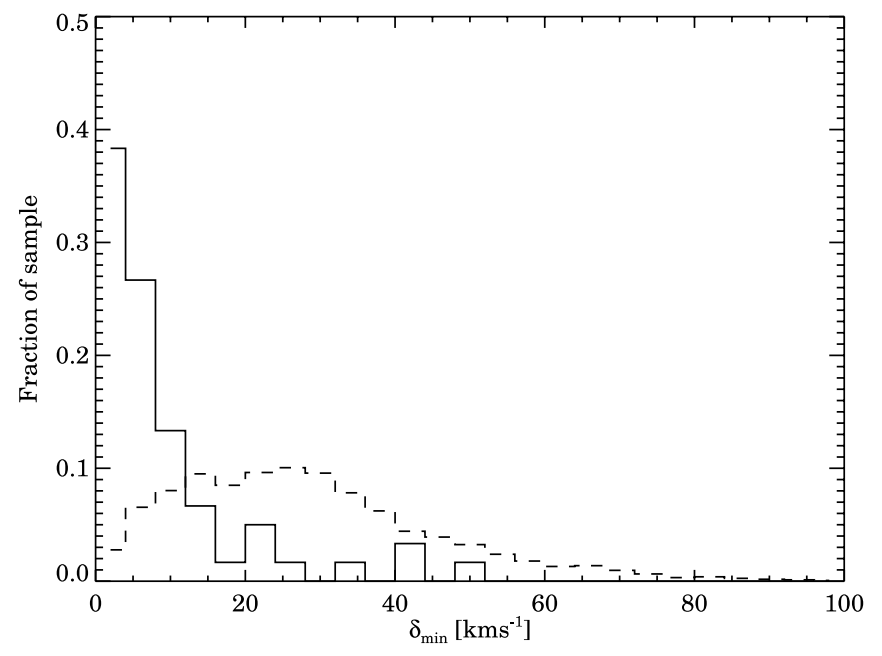

FIG. 3.- Histogram of distances in the three-dimensional velocity space to the closest moving group. Solid line: stars from Table 3; dashed line: volumelimited Hipparcos sample (see $\S 4.1$ ).

associations (see Fig. 2 of Stelzer \& Neuhäuser 2000), yielding an age range of 10-100 Myr. In Table 1 we summarize the age estimates for each object.

\section{DISCUSSION}

\subsection{Connection between Debris Disks and Young Moving Groups}

From our sample of 60 main-sequence stars exhibiting strong infrared excess, 26 can be assigned to young stellar kinematic groups. In order to test whether the frequency of stars belonging to young stellar kinematic groups is similar in a general sample, we determined the corresponding ratio within a volume-limited sample of normal stars. First, we created this sample by selecting stars from the Hipparcos catalog using the following criteria: (1) they are closer than $120 \mathrm{pc}$ (the same volume limit as in our sample), (2) their survey flag in the catalog ${ }^{11}$ was set to " $S$," and (3) they have radial velocity measurement with uncertainty less than $5 \mathrm{~km} \mathrm{~s}^{-1}$. The second condition guarantees that stars observed in various individual projects do not introduce a bias in the analysis of the velocity distribution (Skuljan et al. 1999). In addition, Binney et al. (1997) noted that radial velocities are preferentially observed for high proper motion stars, which may cause a kinematical bias in our sample. Following the proposal of Skuljan et al. (1999), we constrained the sample for stars exhibiting low transverse velocity and excluded all stars with $v_{t} \geq 80 \mathrm{~km} \mathrm{~s}^{-1}$ in order to avoid this bias.

The query resulted in 7519 objects, for which we computed the $U V W$ Galactic space motion components. For each star in the two samples (the 60 debris systems in Table 1 and the newly defined volume-limited stellar sample) we determined the Euclidean distance in the three-dimensional velocity space from the closest moving group, $\delta_{\min }$. In this analysis we considered only groups younger than 150 Myr. In Figure 3 we plotted the histograms of $\delta_{\min }$ for the two samples. A two-sided Kolmogorov-Smirnov test shows that the two distributions are different with a probability higher than $99.99 \%$. This result indicates that debris systems

\footnotetext{
11 Field H68 in the Hipparcos catalog. " $\mathrm{S}$ " indicates that the entry is contained within the "survey," which was the basic list of bright stars added to and merged with the total list of proposed stars, to provide a stellar sample almost complete to well-defined limits. The limiting magnitude was a function of the stars's spectral types and Galactic latitude.
}

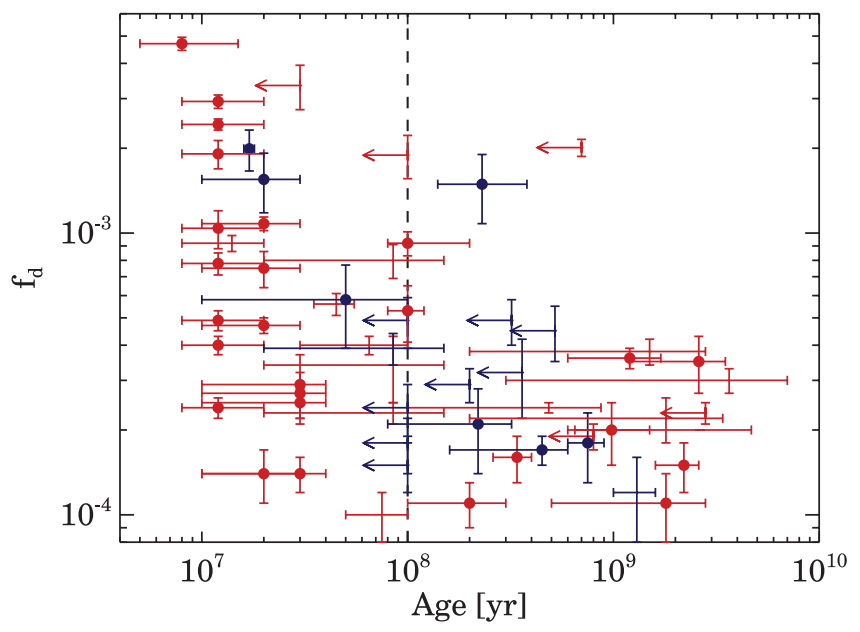

FIG. 4.- Fractional luminosity of the infrared excess as a function of age. Dashed line: threshold of 100 Myr. Upper age limits are denoted by arrows, whose hats correspond to the uncertainty in $f_{d}$. When only an age range is known, no filled circle was plotted. Red symbols mark those debris systems whose existence was explicitely confirmed by an instrument independent of $\operatorname{IRAS}(\S 4.2)$.

of high infrared fractional luminosity are much more intimately linked to the nearby young stellar kinematic groups than the majority of normal stars.

\subsection{The Relationship between Fractional Luminosity and Age}

Zuckerman \& Song (2004b) hypothesized that stars with $f_{d}>$ $10^{-3}$ are younger than $100 \mathrm{Myr}$ and therefore a high $f_{d}$ value can be used as an age indicator. This proposal is in contradiction with the conclusion of Decin et al. (2003), who claimed the existence of high- $f_{d}$ disks around older stars. In order to test which proposal is supported by our data, in Figure 4 we plotted the distribution of ages as a function of the fractional luminosity $f_{d}$ from Table 1. We plotted in red the debris disks whose presence was explicitly confirmed by an instrument independent of IRAS (see col. [8] in Table 1). The confirmation could be based, e.g., on high spatial resolution infrared images (ISO ISOPHOT, Spitzer MIPS), on mid-infrared spectra (Spitzer IRS), or on coronagraphic images (HST ACS, HST NICMOS). We found 43 confirmed debris disks in total.

Most data points with $f_{d}>5 \times 10^{-4}$ fall below the age threshold of 100 Myr (marked by a dashed line), while objects with lower $f_{d}$ show a larger spread in age. This trend can be recognized in the whole sample but is especially clear in the confirmed subsample (red symbols). There is only one noteworthy case: HD 121812 with an age of $230_{-90}^{+150}$ Myr exhibits fractional luminosity exceeding the $5 \times 10^{-4}$ threshold value. However, the presence of a debris disk around this star has not been confirmed independently of IRAS. On the basis of this result, we conclude that, according to the suggestion of Zuckerman \& Song (2004b), the majority of debris disks with $f_{d}>5 \times 10^{-4}$ are younger than $100 \mathrm{Myr}$, and high fractional luminosities can be used as an indicator of youth. Nevertheless, the opposite is not true, i.e., a low $f_{d}$ value is not correlated with age and, in particular, is not an indicator of antiquity.

There is a growing list of debris disk systems that have been discovered by the sensitive detectors of the Spitzer Space Telescope (Beichman et al. 2005a; Bryden et al. 2006; Chen et al. 2005a, 2005b; Kim et al. 2005; Low et al. 2005; Meyer et al. 2004; Stauffer et al. 2005; Uzpen et al. 2005), and one may wonder whether these new observations support our previous 
conclusion. As a preliminary check we collected from the cited papers all debris disks with $f_{d}>5 \times 10^{-4}$. We used fractional luminosity values and age estimates as quoted in the papers. We found that all of these new disks discovered so far belong to the $\sim 16$ Myr old Lower Centaurus Crux subgroup of the ScorpiusCentaurus association (HD 106906, HD 113556, HD 113766, HD 114082, HD 115600, HD 117214; Chen et al. 2005a), to the TWA (TWA 7, TWA 13A, TWA 13B; Low et al. 2005), or to the star-forming region RCW 49 (18 possible warm debris disks; Uzpen et al. 2005), which unambiguously shows that these objects are young, in agreement with the conclusion of the present paper.

\subsection{Debris Disk Evolution and the Cases of Old Systems}

There are a number of models in the literature (see $\S 1$ ) to describe the temporal evolution of debris disks. In the following we compare our results with predictions.

Dominik \& Decin (2003) proposed a simple collisional model that assumes that all dust grains in the debris disk are produced in collisions between planetesimals within a ring whose radius is constant during the whole evolution. In collisional equilibrium, when the dust production and destruction rates are in balance, the grain loss mechanism governs the amount of dust visible in the system. If dust destruction is dominated by collisions, the fractional luminosity $f_{d}$ decreases proportionally to $t^{-1}$. If the dust removal process is dominated by the Poynting-Robertson drag, $f_{d} \propto t^{-2}$. It is predicted that in disks with $f_{d}>10^{-4}$ the evolution is dominated by collisions (Dominik \& Decin 2003; Wyatt 2005).

Three families of disk evolution models, computed from equations (7) and (35)-(40) of Dominik \& Decin (2003), are plotted as shaded bands in Figure 5. The data points and symbols in this figure are identical to those in Figure 4. The main differences between the model families are related to disk mass: (1) $M_{d}=10 M_{\oplus}$, (2) $M_{d}=50 M_{\oplus}$, and (3) $M_{d}=250 M_{\oplus}$. The width of each band corresponds to a range in stellar mass from 0.5 to $3 M_{\odot}$. Additional parameters are the characteristic radius of the ring of planetesimals $r_{c}=43 \mathrm{AU}$, the radius of planetesimals $a_{c}=10 \mathrm{~km}$, the density of the planetesimal material $\rho_{c}=1.5 \mathrm{~g} \mathrm{~cm}^{-3}$ (proposed for icy bodies with a small rocky component; Greenberg 1998; Kenyon 2002), the size of the smallest visible grains $a_{\mathrm{vis}}=10 \mu \mathrm{m}$ (taken from Jura et al. 2004), the absorption efficiency of the dust particles $Q_{\text {abs }}=1$, and $\epsilon_{0}=226$ (defined in eq. [22] of Dominik \& Decin 2003).

Figure 5 shows that the location of most stars on the evolutionary diagram can be explained by the models. The number of older stars exhibiting high $f_{d}$ values incompatible with the models is relatively low. Most of these disks are located in the $t \geq$ $10^{9} \mathrm{yr}$ and $f_{d} \lesssim 5 \times 10^{-4}$ area. A possible explanation for the origin of these stars was proposed by Dominik \& Decin (2003), who assumed that different planetesimal disks become active debris systems at different ages because of the delayed onset of the collisional cascade. Large collisional events may also increase the brightness of a debris disk temporarily (Rieke et al. 2005). Extraordinary events during the evolution like, e.g., a proposed "supercomet" in the HD 69830 system (Beichman et al. 2005b) cannot be excluded either. Nevertheless, the low number of systems incompatible with the models, especially at $f_{d} \gtrsim 5 \times 10^{-4}$, indicates that the above scenarios do not represent the main evolutionary trend.

It is important to note that a large spread in fractional luminosities $\left(10^{-4}<f_{d}<5 \times 10^{-3}\right)$ can be observed in the figure among young debris systems $(t<100 \mathrm{Myr})$. This result resembles the findings of Rieke et al. (2005) among A-type stars but somewhat contradicts those of Decin et al. (2003) and Dominik

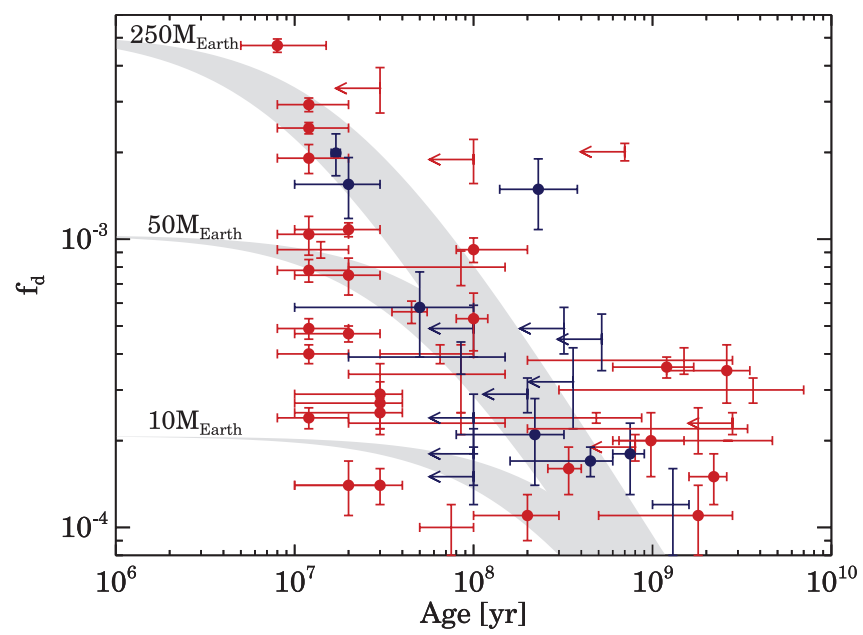

FIG. 5.- Fractional luminosity of the infrared excess as a function of age. The shaded bands mark the evolutionary models of Dominik \& Decin (2003; for detailed model parameters see $\S 4.3$ ). Upper age limits are denoted by arrows, whose hats correspond to the uncertainty in $f_{d}$. When only an age range is known, no filled circle was plotted.

$\&$ Decin (2003), who found only a few young stars with moderate or small infrared excesses and proposed that it might be related to the effect of stirring. A possible explanation of the large spread among young stars could be that the initial conditions of the disks (especially initial disk mass) are far from being homogeneous.

\section{CONCLUSIONS}

We searched the IRAS and ISO databases and compiled a list of debris disks exhibiting the highest fractional luminosity values $\left(f_{d}>10^{-4}\right)$ in the vicinity of the Sun $(d<120 \mathrm{pc})$. Utilizing high-resolution far-infrared maps, we attempted to exclude bogus disks from the sample. We recomputed the fractional luminosity value for each disk using available IRAS, ISO, and Spitzer data and analyzed the Galactic space velocities of the objects, as well as the distribution of the disks on the fractional luminosity versus age diagram. Our results are summarized as follows:

1. We compiled a list of 60 debris disk systems of high fractional luminosity. Eleven of them are new discoveries, and 4 out of these 11 have been confirmed by Spitzer observations.

2. Disks with high fractional luminosity often belong to young stellar kinematic groups, providing an opportunity to obtain improved age estimates for these disks.

3. Practically all objects with $f_{d}>5 \times 10^{-4}$ are younger than $100 \mathrm{Myr}$.

4. The number of old systems with $\operatorname{high} f_{d}$ seems to be lower than was claimed before, mainly as a consequence of the age revision in connection to the young stellar kinematic groups.

5. There exist many young disks of moderate fractional luminosity.

6. Comparing the theoretical evolutionary model of Dominik $\&$ Decin (2003) with the observations in the $f_{d}$ versus age diagram, good general agreement was found.

We are grateful to the anonymous referee for his/her comments that improved the paper.

This research has made use of the IRAS and Hipparcos catalogs (ESA 1997), as well as the SIMBAD database and the VizieR tool operated by CDS, Strasbourg, France. The Two Micron All 
Sky Survey (2MASS) is a joint project of the University of Massachusetts and the Infrared Processing and Analysis Center/ California Institute of Technology, funded by the National Aeronautics and Space Administration and the National Science Foundation.

This work is based on observations made with the Spitzer Space Telescope, which is operated by the Jet Propulsion Laboratory, California Institute of Technology under a contract with NASA. Support for this work was provided by NASA through an award issued by JPL/Caltech.

This material is partly based on work supported by the National Aeronautics and Space Administration through the NASA
Astrobiology Institute under Cooperative Agreement CAN-02OSS-02 issued through the Office of Space Science.

The ISO Data Archive is maintained at the ISO Data Centre, Villafranca, Madrid, and is part of the Science Operations and Data Systems Division of the Research and Scientific Support Department. ISOPHOT observations were reduced using the ISOPHOT Interactive Analysis package PIA, which is a joint development by the ESA Astrophysics Division and the ISOPHOT Consortium, led by the Max-Planck-Institut für Astronomie (MPIA).

The work was partly supported by grants OTKA K62304 and T043739 of the Hungarian Scientific Research Fund.

\section{APPENDIX A}

\section{BOGUS DEBRIS DISKS}

In the last few years several IRAS-based debris disk candidates turned out to be bogus. Examples are HD 155826 (Lisse et al. 2002), or the list of Kalas et al. (2002). The most common problems are contamination by background objects (cirrus knots, galaxies), Pleiades-like nebulosity, or unreliable point-source detection by IRAS. Our list of debris disk candidates is based on IRAS data; however, when higher resolution far-infrared observations were available, we checked whether the above-mentioned problems could have affected the detection of the disk. In Table 6 we list those objects that were identified as debris systems in the literature (and claimed to have $10^{-4}<f_{d}<10^{-2}$ in the original paper) but that our analysis indicates are very likely bogus disks. In the following we briefly describe the reason of rejection.

$H D$ 34739.-The source position in the MIPS $70 \mu \mathrm{m}$ map differs from the star's position by $26^{\prime \prime}$ but coincides with the near-infrared source 2MASS J05163646-5257397 with an offset of $2^{\prime \prime}$.

HD 53842.- This system is not a real bogus disk, since at $24 \mu \mathrm{m}$ the star shows infrared excess (A. Moór et al. 2006, in preparation), but at $70 \mu \mathrm{m}$ the IR emission comes from an independent compact source separated by $19^{\prime \prime}$. This nearby source coincides with 2MASS J06460135-8359359, within a distance of $2^{\prime \prime}$. Due to this fact, the fractional luminosity of HD 53842 decreased below our lower limit.

HD 56099.-The source position in the MIPS $70 \mu \mathrm{m}$ map differs from the star's position by $24^{\prime \prime}$ but coincides with the near-infrared source 2MASS J07190966+5907219 with an offset of $2^{\prime \prime}$.

$H D$ 72390.- The peak brightness position in the ISOPHOT maps at 60 and $90 \mu \mathrm{m}$ differs from the stellar position by $36^{\prime \prime}$. Coordinates of this peak are very close to those of 2MASS J08143635-8423260, which is an extended 2MASS source (XSC 1524951), with an offset of $5^{\prime \prime}$.

TABLE 6

List of Bogus Disks

\begin{tabular}{|c|c|c|c|c|c|c|}
\hline $\begin{array}{l}\text { NAME } \\
(1)\end{array}$ & $\begin{array}{c}\text { Nearby IRAS } \\
\text { Source } \\
\text { (2) }\end{array}$ & $\begin{array}{c}\text { First Reference } \\
\text { as Debris Disk Candidate } \\
\text { (3) }\end{array}$ & $\begin{array}{l}\text { REASON OF } \\
\text { REJECTION } \\
\text { (4) }\end{array}$ & $\begin{array}{c}\text { INSTRUMENT } \\
(5)\end{array}$ & $\begin{array}{l}\text { R.A. } \\
(\mathrm{J} 2000.0) \\
(6)\end{array}$ & $\begin{array}{l}\text { Decl. } \\
(\mathrm{J} 2000.0) \\
(7)\end{array}$ \\
\hline HD 23484 & F03423-3826 & $1^{\mathrm{a}}$ & No detectable excess ${ }^{\mathrm{a}}$ & ISOPHOT & $\ldots$ & $\ldots$ \\
\hline HD 56099 ............................ & F07149+5913 & 1 & Source confusion & MIPS & 071909.6 & +590720 \\
\hline HD 72390 & F08210-8414 & 1 & Source confusion & ISOPHOT & 081439 & -842323 \\
\hline HD 82821 & F09319+0346 & 1 & Source confusion & MIPS & 093436.2 & +033239 \\
\hline HD 143840 & F16001-0440 & 1 & Extended emission & ISOPHOT, MIPS & $\ldots$ & $\ldots$ \\
\hline HD 158373 & F17265-0957 & $1^{\mathrm{a}}$ & No detectable excess ${ }^{\mathrm{a}}$ & ISOPHOT & $\ldots$ & $\ldots$ \\
\hline HD 164330 & F17559+6236 & $1^{\mathrm{a}}$ & No detectable excess ${ }^{\mathrm{a}}$ & ISOPHOT & $\ldots$ & $\ldots$ \\
\hline 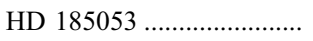 & F19415-8123 & 1 & Extended emission & MIPS & $\ldots$ & $\ldots$ \\
\hline
\end{tabular}

Notes.-Col. (1): Name. Col. (2): Identification of nearby IRAS source. The IRAS source is always located within $30^{\prime \prime}$ of the star position. Col. (3): Reference for first mention as debris disk candidate. Col. (4): Reason why the object was classified as a bogus disk and rejected from further analyses. Col. (5): Instrument. Cols. (6) and (7): Units of right ascension are hours, minutes, and seconds, and units of declination are degrees, arcminutes, and arcseconds. When the object was nominated as a bogus disk due to source confusion, position of the unrelated nearby IR source is given. In the cases of MIPS observations MOPEX was used to extract source coordinates from $70 \mu \mathrm{m}$ MIPS images. The coordinates of peak brightness in ISOPHOT maps were determined by fitting a point-source profile.

${ }^{\text {a }}$ Silverstone (2000) selected these objects as debris disk candidates on the basis of IRAS data, but found that ISOPHOT observations did not confirm the detection of IR excess. He proposed that the nondetection of far-infrared flux excess toward the star's position can be explained by cirrus contamination.

${ }^{\mathrm{b}}$ HD 53842 is not really bogus. At $24 \mu \mathrm{m}$ it shows IR excess above the photosphere (see A. Moór et al. 2006, in preparation). However, at $70 \mu \mathrm{m}$ the excess emission is related to a nearby infrared source.

ReFERENCES.-(1) Silverstone 2000; (2) Mannings \& Barlow 1998. 
TABLE 7

List of Rejected Suspicious Objects

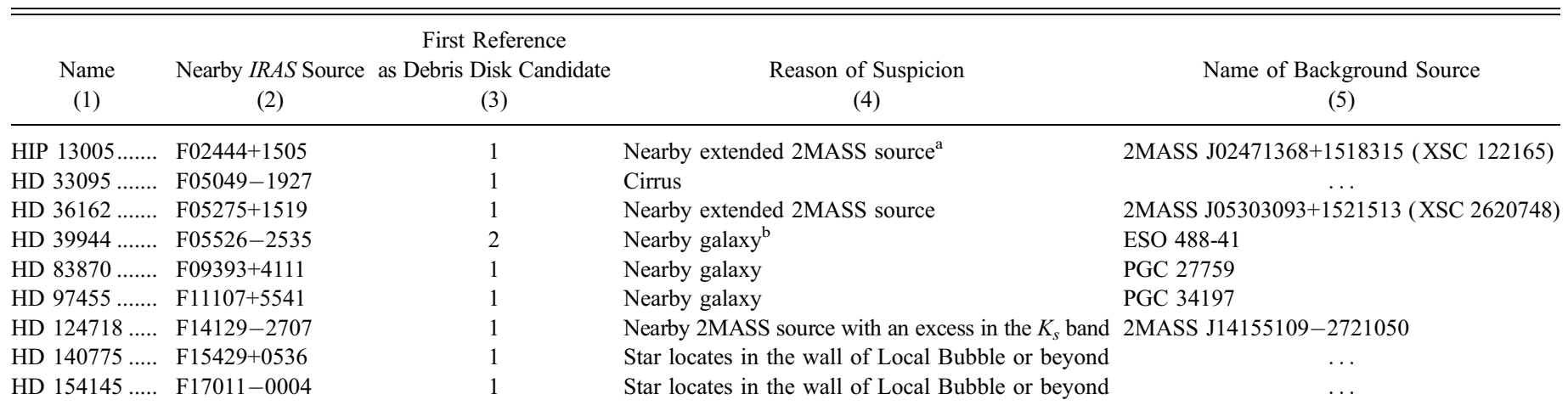

Notes.-Col. (1): Name. Col. (2): Identification of nearby IRAS source. The IRAS source is always located within $30^{\prime \prime}$ of the star position. Col. (3): Reference for first mention as debris disk candidate. Col. (4): Reason of suspicion. For more details see § 2.3. Col. (5): Name of background source.

a Zuckerman \& Song (2004a) also found suspicious this debris candidate based on the offset between its IRAS positions measured at 12 and $60 \mu$ m.

${ }^{\mathrm{b}}$ Sylvester \& Mannings (2000) also noted this coincidence between the position of the IRAS source and the nearby galaxy.

REFERENCES.-(1) Silverstone 2000; (2) Mannings \& Barlow 1998.

HD 82821.-We have detected two infrared sources in the MIPS $70 \mu \mathrm{m}$ map, but their positions significantly differ from the position of the star. The position of the brighter one lies at 70" from the nearby IRAS source (FSC 09319+0346) and is located just outside the $2 \sigma$ error ellipse (nearly along the major axis) but well inside the $3 \sigma$ error ellipse. We assume that this source, whose position coincides well with the near-infrared source 2MASS J09343630+0332417 (XSC 2391850) within a distance of 3 ", is responsible for the source confusion. The second source was probably below the IRAS sensitivity limit.

HD 143840.--ISOPHOT minimap observation at $90 \mu \mathrm{m}$ and a Spitzer MIPS image at $70 \mu \mathrm{m}$ show extended IR emission. The image of the Digitized Sky Survey also shows a reflection nebulosity around this star. We think that the excess far-infrared emission comes from the nebula.

HD 185053.-Magakian (2003) proposed that HD 185053 is the illuminating source of the reflection nebula GN 19.41.5. Spitzer MIPS observations at 24 and $70 \mu \mathrm{m}$ show extended IR emission around the star. We think that the excess far-infrared emission is related to the nebula rather than to a debris disk.

HD 204942.- The source position in the MIPS $70 \mu \mathrm{m}$ map differs from the star's position by $22^{\prime \prime}$ but coincides with the nearinfrared source 2MASS J21323602-2409319 with an offset of 4 ".

$H D$ 23484, HD 158373, and HD 164330.--ISOPHOT minimap observations of these stars at 60 and $90 \mu \mathrm{m}$ did not show excess above the photosphere. This discrepancy between the IRAS-based excesses and the nondetection of excesses by ISOPHOT (which had better spatial resolution than IRAS at far-infrared wavelengths) was already mentioned by Silverstone (2000), who suggested cirrus contamination as the reason.

For stars when no higher resolution data were available a set of criteria were applied to filter out suspicious objects that might be bogus disks (see $\S 2.3$ ). In Table 7 we listed those objects that were earlier identified as debris disks in the literature (with $10^{-4}<$ $f_{d}<10^{-2}$ ) but were rejected from further analysis according to our criteria. Nevertheless, future high spatial resolution infrared data are needed to prove or disprove our judgment.

\section{APPENDIX B}

\section{NEW MEMBERS IN THE YOUNG STELLAR KINEMATIC GROUPS}

$\beta$ Pictoris moving group (BPMG).-We identified two stars that are candidate members of this group: HD 15115 and HD 191089. HD 15115 is a northern object. Although the first surveys found BPMG members only in the southern hemisphere (Barrado y Navascués et al. 1999; Zuckerman et al. 2001a), recently Song et al. (2003) proposed several new northern candidates. One of those, HIP 12545 , is located within $4^{\circ}$ to HD 15115 on the sky. HD 15115 has a ROSAT counterpart with fractional X-ray luminosity of $\log \left(L_{\mathrm{X}} / L_{\mathrm{bol}}\right)=-4.94\left(\log L_{\mathrm{X}}=29.2 \mathrm{ergs} \mathrm{s}^{-1}\right)$. These properties are comparable to those of stars with similar mass in young star associations (see Figs. 9 and 10 of de la Reza \& Pinzón 2004).

HD 191089 is somewhat more distant (see Table 1) than known members of the group in the list of Zuckerman \& Song (2004b). However, a large part of this list is based on a volume-limited survey within $d<50 \mathrm{pc}$, and other authors proposed candidates at larger distances (Torres et al. 2003). HD 191089 shows several signs of youth. Mamajek (2004) classified this star as younger than Pleiades because its lithium abundance $[\mathrm{EW}(\mathrm{Li})=95 \pm 6 \mathrm{~m} \AA$ ] was higher than $95 \%$ of Pleiades stars with similar effective temperatures. Isochronal age of the star (1 $17_{-4}^{+8}$ Myr; Mamajek 2004) is in good agreement with the age of the $\beta$ Pictoris moving group (12 $2_{-4}^{+8} \mathrm{Myr}$; see Table 4). Moreover, HD 191089 rotates rapidly compared with typical F5-type stars ( $v \sin i=45 \mathrm{~km} \mathrm{~s}^{-1}$; Nordström et al. 2004). It has a ROSAT X-ray luminosity of $\log L_{\mathrm{X}}=29.2 \mathrm{ergs} \mathrm{s}^{-1}$ and X-ray fractional luminosity of $\log \left(L_{\mathrm{X}} / L_{\mathrm{bol}}\right)=-4.93$. These values are also comparable with the properties of stars with similar spectral types in nearby kinematic groups (see Figs. 9 and 10 of de la Reza \& Pinzón 2004).

The GAYA2 and TucHor associations.-The Great Austral young association 2 (GAYA2) and Tucana/Horologium (TucHor) association can be discussed together since they overlap both in their location on the projected sky plane and in velocity space. GAYA2 


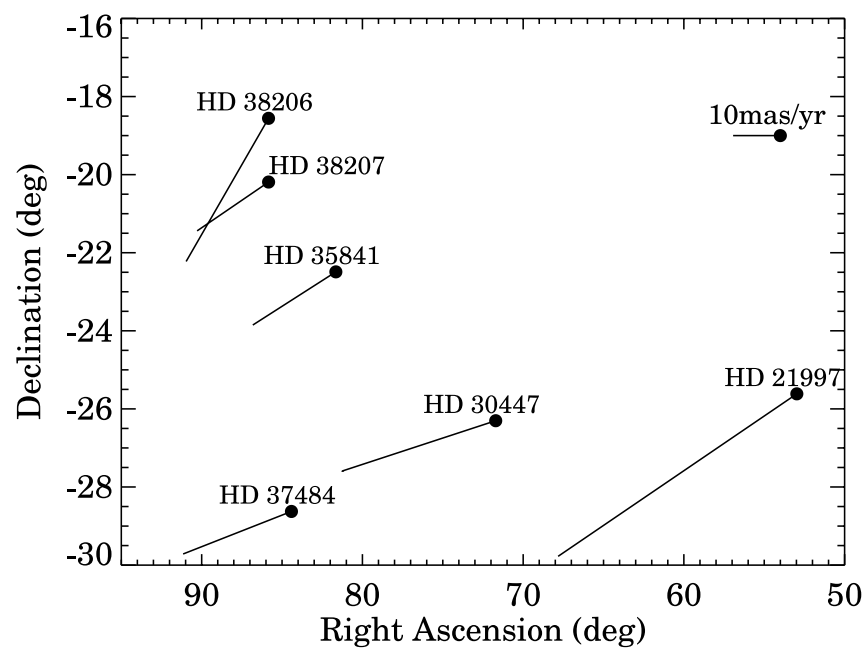

FIG. 6.- Positions and proper motions of proposed new members of the Tucana-Horologium and GAYA2 associations (see Appendix B).

was discovered in the framework of the SACY (Search for Associations Containing Young stars) survey (Torres et al. 2003), and the known members are confined mostly in the right ascension (R.A.) range $3^{\text {h }}<$ R.A. $<9^{\text {h}}$. Recently identified members of TucHor occupy a similar region $\left(2^{\mathrm{h}}<\right.$ R.A. $\left.<7^{\mathrm{h}}\right)$, and several of them show only slightly different Galactic space motions compared to the mean $U V W$ velocities of the Tucana nucleus and resemble the mean space motions of GAYA2. Although GAYA2 is more distant (located at a mean distance of $\sim 84 \mathrm{pc}$, while TucHor members located in the same sky region have a mean distance of $\sim 50 \mathrm{pc}$ ), there is an overlap in radial distance, as well. Studying the relationship between the two associations is beyond the scope of this work. As a practical solution, we assigned all doubtful sources (see Fig. 6) of $D \leq 67 \mathrm{pc}$ to the TucHor association and the more distant ones to GAYA2. Thus, we propose that one of these sources, HD 37484, belongs to TucHor (its space velocity is not inconsistent with that of other neighboring TucHor members). The measured lithium abundance of the star (Favata et al. 1993) is a strong indication of its youth. HD 21997, HD 30447, HD 35841, HD 38206, and HD 38207 are classified as members of the GAYA2 group. It is worth mentioning that these five stars form a spectacular concentration of high- $f_{d}$ debris disks within a relatively small area on the sky.

Local Association.-We propose that HD 10472, HD 10638, HD 218396, and HD 221853 belong to the Local Association. HD 10472 was previously a TucHor candidate (Torres et al. 2000; Zuckerman et al. 2001b), but recently Zuckerman \& Song (2004b) suggested that its membership status is uncertain; thus, it may be consistent with our result.

IC 2391 supercluster.-On the basis of its Galactic space velocity HD 192758 may belong to the IC 2391 supercluster. Its position on the CMD (see Fig. 2 and $\S 3.2 .2$ ) also suggests its youth.

HD 110058.- This was earlier classified as a member of the Lower Centaurus Crux (LCC) association using the convergent point method (de Zeeuw et al. 1999). However, according to our results, its Galactic space velocity is inconsistent with the mean velocity of the LCC. Nevertheless, HD 110058 seems to be a very young object on the basis of its position on the CMD of A-type stars (see $\S 3.2 .2)$.

\section{REFERENCES}

Ábrahám, P., Moór, A., Kiss, Cs., Héraudeau, P., \& del Burgo, C. 2003, in Exploiting the ISO Data Archive: Infrared Astronomy in the Internet Age, ed. C. Gry et al. (ESA SP-511; Noordwijk: ESA), 129

Ardila, D. R., et al. 2004, ApJ, 617, L147

Artymowicz, P. 1996, in The Role of Dust in the Formation of Stars, ed. H. U. Kaüfl \& R. Siebenmorgen (New York: Springer), 137

Aumann, H. H., et al. 1984, ApJ, 278, L23

Backman, D., \& Gillett, F. C. 1987, in Cool Stars, Stellar Systems and the Sun, ed. J. L. Linsky \& R. E. Stencel (Berlin: Springer), 340

Backman, D. E., \& Paresce, F. 1993, in Protostars and Planets III, ed. E. H. Levy \& J. I. Lunine (Tucson: Univ. Arizona Press), 1253

Barrado y Navascués, D. 1998, A\&A, 339, 831

Barrado y Navascués, D., Stauffer, J. R., Song, I., \& Caillault, J.-P. 1999, ApJ, 520, L123

Beichman, C. A., Neugebauer, G., Habing, H. J., Clegg, P. E., \& Chester, T. J., eds. 1988, IRAS Explanatory Supplement (Washington, DC: GPO)

Beichman, C. A., et al. 2005a, ApJ, 622, 1160 2005b, ApJ, 626, 1061

Binney, J. J., Dehnen, W., Houk, N., Murray, C. A., \& Penston, M. J. 1997, in Hipparcos, Venice '97, ed. B. Battrick (ESA SP-402; Noordwijk: ESA), 473 Bryden, G., et al. 2006, ApJ, 636, 1098

Carpenter, J. M., Wolf, S., Schreyer, K., Launhardt, R., \& Henning, T. 2005, AJ, 129, 1049

Chen, C. H., Jura, M., Gordon, K. D., \& Blaylock, M. 2005a, ApJ, 623, 493

Chen, C. H., et al. 2005b, ApJ, 634, 1372
Cutri, R. M., et al. 2003, Explanatory Supplement to the 2MASS All Sky Data Release (Pasadena: IPAC)

Decin, G., Dominik, C., Malfait, K., Mayor, M., \& Waelkens, C. 2000, A\&A, 357,533

Decin, G., Dominik, C., Waters, L. B. F. M., \& Waelkens, C. 2003, ApJ, 598, 636 de la Reza, R., \& Pinzón, G. 2004, AJ, 128, 1812

Dent, W. R. F., Walker, H. J., Holland, W. S., \& Greaves, J. S. 2000, MNRAS, 314,702

de Zeeuw, P. T., Hoogerwerf, R., de Bruijne, J. H. J., Brown, A. G. A., \& Blaauw, A. 1999, AJ, 117, 354

Dominik, C., \& Decin, G. 2003, ApJ, 598, 626

Eggen, O. J. 1989, PASP, 101, 54

ESA. 1997, The Hipparcos and Tycho Catalogues (ESA SP-1200; Noordwijk: ESA)

Evans, D. S. 1967, in IAU Symp. 30, Determination of Radial Velocities and Their Applications, ed. A. H. Batten \& J. F. Heard (London: Academic Press), 57

Favata, F., Barbera, M., Micela, G., \& Sciortino, S. 1993, A\&A, 277, 428

Gabriel, C., Acosta-Pulido, J., Heinrichsen, I., Morris, H., \& Tai, W.-M. 1997, in ASP Conf. Ser. 125, Astronomical Data Analysis Software and Systems VI, ed. G. Hunt \& H. E. Payne (San Francisco: ASP), 108

Gerbaldi, M., Faraggiana, R., Burnage, R., Delmas, F., Gómez, A. E., \& Grenier, S. 1999, A\&AS, 137, 273

Girardi, L., Bressan, A., Bertelli, G., \& Chiosi, C. 2000, A\&AS, 141, 371

Gordon, K. D., et al. 2005, PASP, 117, 503

Greaves, J. S., Mannings, V., \& Holland, W. S. 2000, Icarus, 143, 155 
Greenberg, J. M. 1998, A\&A, 330, 375

Grenier, S., et al. 1999, A\&AS, 137, 451

Habing, H. J., et al. 2001, A\&A, 365, 545

Harper, D. A., Loewenstein, R. F., \& Davidson, J. A. 1984, ApJ, 285, 808

Heinrichsen, I., Walker, H. J., Klaas, U., Sylvester, R. J., \& Lemke, D. 1999, MNRAS, 304, 589

Helou, G., \& Walker, D. W., eds. 1988, IRAS Catalogs: The Small Scale Structure Catalog (Washington, DC: GPO)

Hines, D. C., et al. 2005, FEPS Data Explanatory Supplement, Version 3.0 (Pasadena: SSC)

Holland, W., et al. 1998, Nature, 392, 788

Jarrett, T. H., Chester, T., Cutri, R., Schneider, S., \& Huchra, J. P. 2000, AJ, 119,2498

Jura, M. 1991, ApJ, 383, L79

Jura, M., White, R., Telesco, C., Pina, R., \& Fisher, R. S. 1998, ApJ, 505, 897

Jura, M., et al. 2004, ApJS, 154, 453

Kalas, P. 2005, ApJ, 635, L169

Kalas, P., Graham, J. R., Beckwith, S. V. W., Jewitt, D. C., \& Lloyd, J. P. 2002, ApJ, 567, 999

Kenyon, S. J. 2002, PASP, 114, 265

Kenyon, S. J., \& Bromley, B. C. 2002, ApJ, 577, L35 2004, AJ, 127, 513

Kessler, M. F., et al. 1996, A\&A, 315, L27

Kharchenko, N. V., Piskunov, A. E., \& Scholz, R.-D. 2004, Astron. Nachr., 325,439

Kim, J. S, et al. 2005, ApJ, 632, 659

King, J. R., Villarreal, A. R., Soderblom, D. R., Gulliver, A. F., \& Adelman, S. J. 2003, AJ, 125, 1980

Kleinmann, S. G., Cutri, R. M., Young, E. T., Low, F. J., \& Gillett, F. C. 1986, Explanatory Supplement to the IRAS Serendipitous Survey Catalog (Pasadena: JPL)

Koerner, D. W., Ressler, M. E., Werner, M. W., \& Backman, D. E. 1998, ApJ, 503, L83

Krist, J. E., et al. 2005, AJ, 129, 1008

Lachaume, R., Dominik, C., Lanz, T., \& Habing, H. J. 1999, A\&A, 348, 897

Lallement, R., Welsh, B. Y., Vergely, J. L., Crifo, F., \& Sfeir, D. 2003, A\&A, 411,447

Laureijs, R. J., Klaas, U., Richards, P. J., Schulz, B., \& Ábrahám, P. 2003, The ISO Handbook, Vol. 4: PHT-The Imaging Photo-Polarimeter, Version 2.0.1 (ESA SP-1262; Noordwijk: ESA)

Leggett, S. K. 1992, ApJS, 82, 351

Lemke, D., et al. 1996, A\&A, 315, L64

Lisse, C., et al. 2002, ApJ, 570, 779

Liu, M. C., Matthews, B. C., Williams, J. P., \& Kalas, P. G. 2004, ApJ, 608, 526

Low, F. J., Smith, P. S., Werner, M., Chen, C., Krause, V., Jura, M., \& Hines, D. C. 2005, ApJ, 631, 1170

Lowrance, P. J., et al. 2000, ApJ, 541, 390

Luhman, K. L., Stauffer, J. R., \& Mamajek, E. E. 2005, ApJ, 628, L69

Magakian, T. Y. 2003, A\&A, 399, 141

Makovoz, D., \& Marleau, F. 2005, PASP, 117, 1113

Mamajek, E. E. 2004, Ph.D. thesis, Univ. Arizona

Mamajek, E. E., Meyer, M. R., Hinz, P. M., Hoffmann, W. F., Cohen, M., \& Hora, J. L. 2004, ApJ, 612, 496

Mamajek, E. E., Meyer, M. R., \& Liebert, J. 2002, AJ, 124, 1670

Mannings, V., \& Barlow, M. 1998, ApJ, 497, 330

Meyer, M. R., et al. 2004, ApJS, 154, 422

Meynet, G., Mermilliod, J.-C., \& Maeder, A. 1993, A\&AS, 98, 477

Montes, D., et al. 2001, MNRAS, 328, 45

Moshir, M., et al. 1989, Explanatory Supplement to the IRAS Faint Source Survey (Pasadena: JPL)
Nordström, B., et al. 2004, A\&A, 418, 989

Oudmaijer, R. D., van der Veen, W. E. C. J., Waters, L. B. F. M., Trams, N. R.,

Waelkens, C., \& Engelsman, E. 1992, A\&AS, 96, 625

Patten, B. M., \& Willson, L. A. 1991, AJ, 102, 323

Plets, H., \& Vynckier, C. 1999, A\&A, 343, 496

Rebull, L. M., Stapelfeldt, K. R., Chen, C., Werner, M. W., Song, I., \&

Zuckerman, B. 2004, BAAS, 205, 17.03

Rieke, G. H., et al. 2004, ApJS, 154, 25

. 2005, ApJ, 620, 1010

Rocha-Pinto, H. J., Flynn, C., Scalo, J., Hänninen, J., Maciel, W. J., \& Hensler, G. 2004, A\&A, 423, 517

Sadakane, K., \& Nishida, M. 1986, PASP, 98, 685

Sartori, M. J., Lépine, J. R. D., \& Dias, W. D. 2003, A\&A, 404, 913

Schneider, G., Silverstone, M. D., \& Hines, D. C. 2005, ApJ, 629, L117

Schneider, G., et al. 1999, ApJ, 513, L127

Silverstone, M. D. 2000, Ph.D. thesis, UCLA

Skuljan, J., Hearnshaw, J. B., \& Cottrell, P. L. 1999, MNRAS, 308, 731

Smith, B., \& Terrile, R. 1984, Science, 226, 1421

Song, I., Caillault, J.-P., Barrado y Navascués, D., \& Stauffer, J. R. 2001, ApJ, 546,352

Song, I., Caillault, J.-P., Barrado y Navascués, D., Stauffer, J. R., \& Randich, S. 2000, ApJ, 533, L41

Song, I., Zuckerman, B., \& Bessell, M. S. 2003, ApJ, 599, 342

2004, ApJ, 614, L125

Spangler, C., Sargent, A. I., Silverstone, M. D., Becklin, E. E., \& Zuckerman, B. 2001, ApJ, 555, 932

Stauffer, J. R., Schultz, G., \& Kirkpatrick, J. D. 1998, ApJ, 499, L199

Stauffer, J. R., et al. 2005, AJ, 130, 1834

Stelzer, B., \& Neuhäuser, R. 2000, A\&A, 361, 581

Stencel, R. E., \& Backman, D. E. 1991, ApJS, 75, 905

Strassmeier, K., Washuettl, A., Granzer, Th., Scheck, M., \& Weber, M. 2000, A\&AS, 142, 275

Sylvester, R., \& Mannings, V. 2000, MNRAS, 313, 73

Thi, W. F., et al. 2001, ApJ, 561, 1074

Torres, C. A. O., da Silva, L., Quast, G. R., de la Reza, R., \& Jilinski, E. 2000, AJ, 120,1410

Torres, C. A. O., Quast, G. R., de la Reza, R., da Silva, L., \& Melo, C. H. F. 2003, in ASP Conf. Ser. 287, Galactic Star Formation across the Stellar Mass Spectrum, ed. J. M. De Buizer \& N. S. van der Bliek (San Francisco: ASP), 439

Uzpen, B., et al. 2005, ApJ, 629, 512

Walker, H. J., \& Heinrichsen, I. 2000, Icarus, 143, 147

Webb, R. A., Zuckerman, B., Platais, I., Patience, J., White, R. J., Schwartz, M. J., \& McCarthy, C. 1999, ApJ, 512, L63

Weintraub, D. A., Saumon, D., Kastner, J. H., \& Forveille, T. 2000, ApJ, 530, 867

Werner, M. W., et al. 2004, ApJS, 154, 1

Williams, J. P., Najita, J., Liu, M. C., Bottinelli, S., Carpenter, J. M., Hillenbrand,

L. A., Meyer, M. R., \& Soderblom, D. R. 2004, ApJ, 604, 414

Wilson, R. E. 1953, General Catalogue of Stellar Radial Velocities (Washington,

DC: Carnegie Inst. Washington)

Wright, C. O., Egan, M. P., Kraemer, K. E., \& Price, S. D. 2003, AJ, 125, 359 Wright, J. T., Marcy, G. W., Butler, R. P., \& Vogt, S. S. 2004, ApJS, 152, 261 Wyatt, M. C. 2005 , A\&A, 433, 1007

Zuckerman, B. 2001, ARA\&A, 39, 549

Zuckerman, B., \& Song, I. 2004a, ApJ, 603, 738

.2004b, ARA\&A, 42, 685

Zuckerman, B., Song, I., Bessel, M. S., \& Webb, R. A. 2001a, ApJ, 562, L87

Zuckerman, B., Song, I., \& Webb, R. A. 2001b, ApJ, 559, 388

Zuckerman, B., \& Webb, R. A. 2000, ApJ, 535, 959 